\title{
Rare two-body decays of the top quark into a bottom meson plus an up or charm quark
}

\author{
David d'Enterria $^{a}$ and Hua-Sheng Shao ${ }^{b}$ \\ ${ }^{a}$ CERN, EP Department, \\ CH-1211 Geneva 23, Switzerland \\ ${ }^{b}$ Laboratoire de Physique Théorique et Hautes Energies (LPTHE), \\ UMR 7589, Sorbonne Université et CNRS, \\ 4 place Jussieu, 75252 Paris Cedex 05, France \\ E-mail: david.d'enterria@cern.ch, huasheng.shao@lpthe.jussieu.fr
}

ABSTRACT: Rare two-body decays of the top quark into a neutral bottom-quark meson plus an up- or charm-quark: $t \rightarrow \bar{B}^{0}+u, c ; t \rightarrow \bar{B}_{s}^{0}+c, u$; and $t \rightarrow \Upsilon(n S)+c, u$, are studied for the first time. The corresponding partials widths are computed at leading order in the non-relativistic QCD framework. The sums of all two-body branching ratios amount to $\mathcal{B}\left(t \rightarrow \bar{B}^{0}+\right.$ jet $) \approx \mathcal{B}\left(t \rightarrow \bar{B}_{s}^{0}+\right.$ jet $) \approx 4.2 \cdot 10^{-5}$ and $\mathcal{B}(t \rightarrow \Upsilon(n S)+$ jet $) \approx 2 \cdot 10^{-9}$, respectively. The feasibility to observe the $t \rightarrow \bar{B}_{(s)}^{0}+$ jet decay is estimated in toppair events produced in proton-proton collisions at $\sqrt{s}=14,100 \mathrm{TeV}$ at the LHC and FCC, respectively. Combining many exclusive hadronic $\bar{B}_{(s)}^{0}$ decays, with $J / \psi$ or $D^{0, \pm}$ final states, about 50 (16000) events are expected in $3(20) \mathrm{ab}^{-1}$ of integrated luminosity at the LHC (FCC), after typical selection criteria, acceptance, and efficiency losses. An observation of the two-body top-quark decay can also be achieved in the interesting $t \rightarrow$ $b($ jet $)+c($ jet $)$ dijet final state, where the $\bar{B}_{(s)}^{0}$ decay products are reconstructed as a jet, with 5300 and 1.4 million signal events above backgrounds expected after selection criteria at the LHC and FCC, respectively. Such unique final states provide a new direct method to precisely measure the top-quark mass via simple 2-body invariant mass analyses.

KeYwords: QCD Phenomenology

ARXIV EPRINT: 2005.08102 


\section{Contents}

1 Introduction 1

2 Theoretical calculations 2

2.1 Top decays into a $\bar{B}_{(s)}^{0}$-meson plus a $u$ - or $c$-quark 4

2.2 Top decays into an $\Upsilon(n S)$-meson plus a $u$ - or $c$-quark 5

3 Measurement of $t \rightarrow \bar{B}_{(s)}^{0}+$ jet in pp collisions at the LHC and FCC 8

3.1 Expected top-quark yields 9

$\begin{array}{lll}3.1 .1 & \text { Fully hadronic } t \bar{t} \text { final state } & 10\end{array}$

$\begin{array}{lll}3.1 .2 & \text { Lepton }+ \text { jets } t \bar{t} \text { final state } & 11\end{array}$

3.2 Measurement of the $t \rightarrow \bar{B}_{(s)}^{0}+$ jet decay $\quad 12$

$\begin{array}{lll}3.2 .1 & \text { Final states with } J / \psi \text { mesons } & 12\end{array}$

$\begin{array}{lll}3.2 .2 & \text { Final states with } D^{0, \pm} \text { mesons } & 13\end{array}$

4 Measurement of $t \rightarrow b($ jet $)+c($ jet $)$ in pp collisions at the LHC and FCC 16

$\begin{array}{lll}4.1 & \text { Top-quark mass via bottom+charm dijet decays } 20\end{array}$

$\begin{array}{lll}5 & \text { Summary } & 21\end{array}$

\section{Introduction}

The top quark decays with $\sim 100 \%$ probability into a $W^{ \pm}$boson plus a $b$-quark, $t \rightarrow W b$. The $W^{ \pm}$boson itself decays in about $2 / 3$ of the cases into hadrons, via the CabibboKobayashi-Maskawa (CKM) enhanced channels ${ }^{1} W^{+} \rightarrow u \bar{d}, c \bar{s}$ (proportional to $V_{u d, c s}=$ 0.974 ), and much less so via the CKM-suppressed $W^{+} \rightarrow u \bar{s}, c \bar{d}$ (with $V_{u s, c d}=0.225$ ) and $W^{+} \rightarrow c \bar{b}, u \bar{b}$ (with $V_{c b}, V_{u b} \approx 0.04,0.004$ ) modes [1]. In this work, we consider the process where one of the down-type quarks $(d, s, b)$ from the $W^{ \pm}$decay subsequently recombines with the $b$-quark coming directly from the top decay to form a $\bar{B}^{0}$ - or $\Upsilon$-meson, thereby leading to the two-body final-states shown in figure 1. To our knowledge, such decays have never been studied in the literature so far. If such top-quark decays have an experimentally visible branching ratio, they can be used e.g. to derive an independent value of the top mass $\left(m_{t}\right)$ through a simple invariant mass analysis of $\bar{B}_{(s)^{-}}^{0}($ or $\Upsilon)$-plus-jet pairs around $m_{t}$. The top mass is one of the key parameters of the Standard Model (SM), chiefly affecting theoretical predictions of Higgs boson properties and searches for new physics beyond the SM (BSM), as well as playing a leading role in the stability of the electroweak vacuum at asymptotic energies [2]. Having at hand different methods to determine $m_{t}$,

\footnotetext{
${ }^{1}$ Hereafter, unless explicitly stated otherwise, the $t \rightarrow W^{+} b \rightarrow \bar{B}_{(s)}^{0}+X$ decays quoted by default are meant to represent also the equivalent $\bar{t} \rightarrow W^{-} b \rightarrow B_{(s)}^{0}+X$ charge-conjugate decays.
} 


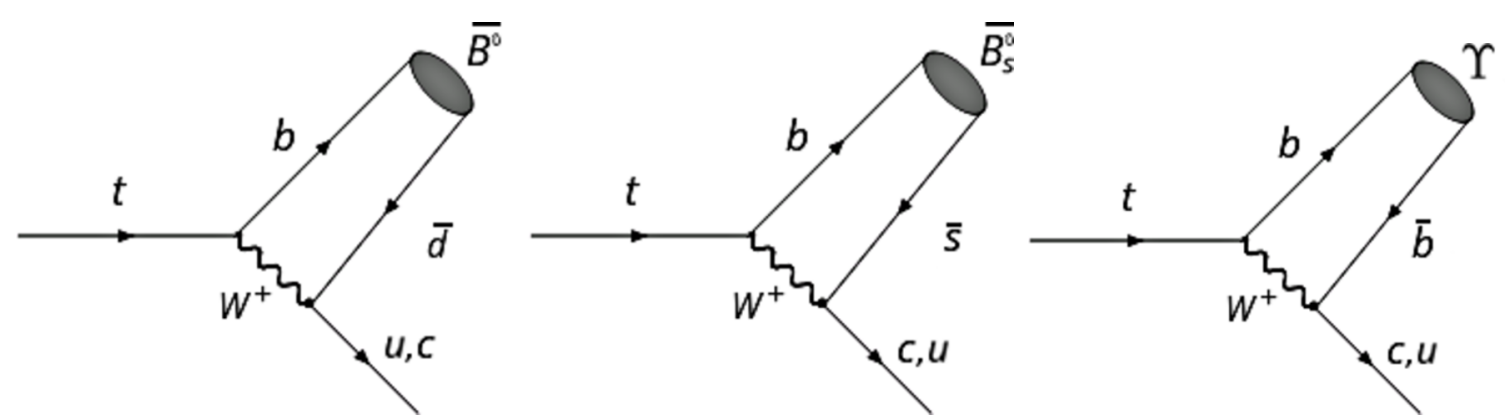

Figure 1. Tree-level Feynman diagrams for the two-body top-quark decays $t \rightarrow \bar{B}^{0}+u, c$ (left), $t \rightarrow \bar{B}_{s}^{0}+c, u$ (center), and $t \rightarrow \Upsilon+c, u$ (right). The quark ordering in the labels $(u, c$ or $c, u$ ) indicates the hierarchy of most probable decays, as computed hereafter.

and thereby precisely derive its value, is essential for testing the overall SM consistency, reducing parametric uncertainties in the extraction of other various key SM parameters, and constraining BSM models through precision electroweak fits.

Rare top-quark decays can be measured by exploiting the very large top-quark data samples expected to be collected in proton-proton (pp) collisions at centre-of-mass (c.m.) energies of $\sqrt{s}=14 \mathrm{TeV}$ during the high-luminosity phase of the Large Hadron Collider (HL-LHC) [3], as well as at $\sqrt{s}=100 \mathrm{TeV}$ at the planned Future Circular Collider (FCC) $[4,5]$. At the HL-LHC, the ATLAS and CMS experiments are expected to integrate luminosities of the order of $\mathcal{L}_{\text {int }}=300 \mathrm{fb}^{-1}$ per year, adding up to $3 \mathrm{ab}^{-1}$ over the projected lifetime of ten years, whereas the FCC-pp plans to integrate $20 \mathrm{ab}^{-1}$ over twenty years of operation. For the $\sim 1$ (35) nb inclusive top-pair cross section expected at $\sqrt{s}=14$ (100) $\mathrm{TeV}[5,6]$, the experiments would thereby be able to analyse about $6 \cdot 10^{9}\left(1.5 \cdot 10^{12}\right)$ top-plus-antitop quark decays for rare searches such as the one under consideration here.

In this paper, we compute first the branching ratios for the two-body decays shown in figure 1 making use of the non-relativistic QCD framework to describe the $(b \bar{d}),(b \bar{s})$, and $(b \bar{b})$ bound-state formation from the top-quark decay products (section 2). A detailed study of the experimental feasibility of measuring such rare two-body decays at the LHC and FCC is described in section 3, in many different $\bar{B}_{(s)}^{0}$ decay modes. Section 4 discusses the observation of the top-quark 2-body decay into bottom +charm jets, including estimates of the expected precision on $m_{t}$ based on $b+c$ dijet invariant mass analyses. The paper ends with a summary of the main results in section 5 .

\section{Theoretical calculations}

The production of a $B$ meson from a $b$-quark, besides the well-known $b \rightarrow B$ parton fragmentation process, can also proceed through a quark recombination mechanism [7] whereby a lighter $d$ or $s$ antiquark from the same hard scattering that produces the bottom quark combines with the latter to form a $\bar{B}^{0} \equiv(b \bar{d})$ or $\bar{B}_{s}^{0} \equiv(b \bar{s})$ bound state. In such cases, the momentum of the $B$ meson arises from the combination of the down or strange plus bottom quark momenta, where both quarks are moving along the same direction. 
To better understand the process, it is informative to consider first the kinematics of such a two-body decay in more detail. In order to form a bound state $B$, in the nonrelativistic limit [8] the four-momenta $P^{\mu}$ of the $B$ meson and of the $b$ and $\bar{q}$ quarks in figure 1 have to satisfy:

$$
P_{b}^{\mu}=\frac{m_{b}}{m_{b}+m_{\bar{q}}} P_{B}^{\mu}, \quad P_{\bar{q}}^{\mu}=\frac{m_{\bar{q}}}{m_{b}+m_{\bar{q}}} P_{B}^{\mu} .
$$

From this, momentum conservation and on-shell relationships, it follows that the invariant mass of the quarks from the $W \rightarrow q^{\prime} \bar{q}$ decay must be of the order of $\mathcal{O}\left(m_{t} \sqrt{m_{\bar{q}} / m_{b}}\right) \approx$ $45 \mathrm{GeV}$, i.e. the $W$ boson is off-shell with $\left|m_{W^{*} \rightarrow q^{\prime} \bar{q}}-m_{W}\right| \gg \Gamma_{W} \approx 2 \mathrm{GeV}$. In the rest frame of the top quark, the leading-order (LO) kinematics of the two-body decay leads to the following typical momenta of the $\bar{B}_{(s)}^{0}$ meson and back-to-back quark $q$ :

$$
\begin{aligned}
p_{B} & =\left\{\varepsilon\left(m_{t}, m_{B}, m_{q}\right), \pi\left(m_{t}, m_{B}, m_{q}\right) \vec{e}\right\}, \\
p_{q} & =\left\{\varepsilon\left(m_{t}, m_{q}, m_{B}\right),-\pi\left(m_{t}, m_{B}, m_{q}\right) \vec{e}\right\},
\end{aligned}
$$

where $\vec{e}$ is a unity three-dimensional vector $|\vec{e}|=1$ and

$$
\begin{aligned}
& \pi\left(m_{t}, m_{B}, m_{q}\right)=\frac{1}{2 m_{t}} \sqrt{m_{t}^{4}+m_{B}^{4}+m_{q}^{4}-2 m_{t}^{2} m_{B}^{2}-2 m_{t}^{2} m_{q}^{2}-2 m_{B}^{2} m_{q}^{2}} \approx \frac{m_{t}}{2} \\
& \varepsilon\left(m_{t}, m_{B}, m_{q}\right)=\frac{m_{t}^{2}+m_{B}^{2}-m_{q}^{2}}{2 m_{t}} \approx \frac{m_{t}}{2} .
\end{aligned}
$$

With the replacement $q \rightarrow W$ in the expressions above, one can see that the bottom quark shares more momentum in the two-body decays of figure 1 than in the standard top-quark final state where the $W$ boson is on-shell. In this latter case, the energies of the $W$-decay quarks are typically $\left(m_{t}^{2}+m_{W}^{2}\right) /\left(4 m_{t}\right) \approx 50 \mathrm{GeV}$, whereas in the two-body decay the uptype quark from the $t \rightarrow \bar{B}^{0} q$ decay carries an energy of the order of $m_{t} / 2$ and is thereby more boosted than in the common $t \rightarrow b W \rightarrow b q^{\prime} \bar{q}$ channel.

For the formation of a bound state of a heavy quark and an antiquark, the nonrelativistic quantum chromodynamics (NRQCD) approach [8], in which the quark interactions are organized in an expansion in $v$ (the typical relative velocity of the quarks inside the meson state) provides a useful theoretical framework. The relevant partial width calculations are quite analogous to those for $B_{c}^{ \pm}$production from final-state $b$ and $c$ quarks in NRQCD [7, 9]. For a decay process $a \rightarrow \bar{B}+X$, the partial width $\Gamma$ reads

$$
d \Gamma[a \rightarrow \bar{B}+X]=\sum_{n} d \hat{\Gamma}\left[a \rightarrow(b \bar{q})_{n}+X\right] \rho\left[(b \bar{q})_{n} \rightarrow \bar{B}\right],
$$

where $(b \bar{q})_{n}$ represents a given Fock state $n$ of the bottom $b$ and light- $\bar{q}$ quarks, and the factor $\rho\left[(b \bar{q})_{n} \rightarrow \bar{B}\right]$ is a non-perturbative probability for $(b \bar{q})_{n}$ to evolve into the $\bar{B}$ meson. However, unlike in the case of $B_{c}^{ \pm}$production, the non-perturbative transition $(b \bar{q})_{n} \rightarrow \bar{B}$ does not follow a definite velocity power-counting because the relative velocity of the light $\bar{d}$ or $\bar{s}$ quarks in the rest frame of the $B$ meson is not small. Therefore, Fock state $(b \bar{q})_{n}$ contributions to the $\bar{B}$ meson with different quantum numbers (e.g. colour or angular momentum) are not necessarily suppressed. 
In ref. [7], it was argued that one only needs to consider Fock states with the $S$ wave orbital angular momentum, because higher orbital states are suppressed by at least a factor of $m_{b} / \Lambda_{\mathrm{QCD}} \approx 20$. In this case, the only relevant states are $n={ }^{1} S_{0}^{[1]},{ }^{3} S_{1}^{[1]},{ }^{1} S_{0}^{[8]},{ }^{3} S_{1}^{[8]}$, where we use the spectroscopic notation ${ }^{2 s+1} L_{J}^{[c]}$ with $s$ being spin, $L$ being orbital angular momentum, $J$ being total angular momentum, and $c=1,8$ being the colour singlet and octet representations, respectively. By using the heavy-quark spin symmetry [8], we can further reduce the non-perturbative transition probabilities $\rho$, from four to two, i.e.

$$
\begin{aligned}
\rho_{1}^{\bar{B}} & \equiv \rho\left[(b \bar{q})_{{ }_{1} S_{0}^{[1]}} \rightarrow \bar{B}\right], \\
\rho_{8}^{\bar{B}} & \equiv \rho\left[(b \bar{q})_{{ }_{S_{0}}^{[8]}} \rightarrow \bar{B}\right], \\
3 \rho_{1,8}^{\bar{B}} & =\rho\left[(b \bar{q})_{{ }_{3} S_{1}^{[1,8]}} \rightarrow \bar{B}\right] .
\end{aligned}
$$

As shown in ref. [7], the $\rho_{1}$ and $\rho_{8}$ probabilities can then be derived from the standard longdistance matrix elements (LDMEs), $\left\langle\mathcal{O}^{\bar{B}}\left({ }^{2 s+1} L_{J}^{[c]}\right)\right\rangle$, widely used in quarkonium physics:

$$
\begin{aligned}
\left\langle\mathcal{O}^{\bar{B}}\left({ }^{1} S_{0}^{[1]}\right)\right\rangle & =2 N_{c} \frac{4 m_{b} m_{\bar{q}}^{2}}{3} \rho_{1,8}^{\bar{B}}, \\
\left\langle\mathcal{O}^{\bar{B}}\left({ }^{1} S_{0}^{[8]}\right)\right\rangle & =\left(N_{c}^{2}-1\right) \frac{4 m_{b} m_{\bar{q}}^{2}}{3} \rho_{1,8}^{\bar{B}}, \\
\left\langle\mathcal{O}^{\bar{B}}\left({ }^{3} S_{1}^{[1,8]}\right)\right\rangle & =3\left\langle\mathcal{O}^{\bar{B}}\left({ }^{1} S_{0}^{[1,8]}\right)\right\rangle,
\end{aligned}
$$

where $m_{b}$ is the bottom quark mass, $m_{\bar{q}}=m_{\bar{d}}, m_{\bar{s}} \approx 0.3 \mathrm{GeV}$ are the constituent light quark masses, and $N_{c}=3$ the number of colours.

So far the discussion focused on the top decays into $\bar{B}_{(s)}^{0}+$ jets. In the case of top decays leading to bottomonium states plus a jet, $t \rightarrow \Upsilon(n S)+c, u$ (figure 1 right), the two bottom quarks from the $W$ and $t$ decays recombine with relative velocity $v \simeq \sqrt{0.1}$ (as given from the $\Upsilon(n S)$ binding energies) [8], the NRQCD velocity scaling rule is well applicable, and various orbital states can contribute. However, as discussed below, such a decay mode is reduced by at least a factor of $\left|V_{c b, u b}\right|^{2} /\left|V_{u d, c s}\right|^{2} \approx 10^{-4}$ compared to the $\bar{B}_{(s)}^{0}+$ jet ones, mostly due to the already very-suppressed decays of $W$ bosons into bottom quarks.

\subsection{Top decays into a $\bar{B}_{(s)}^{0}$-meson plus a $u$ - or $c$-quark}

We compute first the top decay modes shown in the left and center plots of figure 1. Based on the NRQCD recombination framework described above, the LO (in the QCD coupling $\alpha_{s}$ ) partial widths can be obtained from the following expressions

$$
\begin{aligned}
& \Gamma\left(t \rightarrow \bar{B}^{0}+q+X\right)=\left(\rho_{1}^{\bar{B}}+8 \rho_{8}^{\bar{B}}\right)\left|V_{q d}\right|^{2} \frac{\alpha^{2} \pi}{9} \frac{m_{\bar{d}}^{2}\left(m_{t}^{2}-m_{b}^{2}\right)^{2}\left(m_{t}^{2}+m_{b}^{2}\right)}{m_{t}^{3} m_{W}^{4} \sin ^{4} \theta_{w}}\left[1+\mathcal{O}\left(\frac{m_{q}}{m_{b}}\right)\right], \\
& \Gamma\left(t \rightarrow \bar{B}_{s}^{0}+q+X\right)=\frac{\left|V_{q s}\right|^{2}}{\left|V_{q d}\right|^{2}} \frac{m_{\bar{s}}^{2}}{m_{\bar{d}}^{2}} \Gamma\left(t \rightarrow \bar{B}^{0}+q+X\right),
\end{aligned}
$$

with the various input parameters listed in table 1 . Here, we write the generic $t \rightarrow \bar{B}_{(s)}^{0}+$ $q+X$ decay that includes not only exclusive two-body $t \rightarrow \bar{B}_{(s)}^{0}+q$ decays, but also semiinclusive ones from colour octet configurations, those with $c=8$ in eq. (2.5), where $X$ is a 


\begin{tabular}{|cl|cl|}
\hline Masses (and top width) & & Other SM parameters & \\
\hline$m_{d}$ & $0.3 \mathrm{GeV}$ & $\sin \theta_{w}$ & 0.471423 \\
$m_{s}$ & $0.3 \mathrm{GeV}$ & $\alpha^{-1}$ & 132.0 \\
$m_{c}$ & $1.5 \mathrm{GeV}$ & $\alpha_{s}$ & 0.1180 \\
$m_{b}$ & $4.75 \mathrm{GeV}$ & $\left|V_{t b}\right|$ & 0.99 \\
$m_{t}$ & $173.0 \mathrm{GeV}$ & $\left|V_{u d, c s}\right|$ & 0.974 \\
$m_{W}$ & $80.419 \mathrm{GeV}$ & $\left|V_{u s, c d}\right|$ & 0.225 \\
& & $\left|V_{c b}\right|$ & 0.04 \\
$\Gamma_{t}$ & $1.35 \mathrm{GeV}$ & $\left|V_{u b}\right|$ & 0.004 \\
\hline
\end{tabular}

Table 1. Numerical values of the SM parameters used in the calculations.

soft gluon radiated in the transition into the final colourless $B$ hadron. These soft gluons hadronize into low-energy pions or kaons with energies close to the binding energy of the $B$ hadron $\left(\Delta E \approx 400 \mathrm{MeV}\right.$ in the rest frame of the initial $(b \bar{q})_{n}$ Fock state). To evaluate numerically the expressions of eq. (2.6), we use the parameters listed in table 1 , with the $\rho_{1,8}^{\bar{B}}$ transition probabilities estimated as in ref. [7] by relating them to the $\rho_{1,8}^{D}$ ones, where the latter are determined from the $D^{*}$-meson fragmentation function calculation of ref. [10]. Assuming $\rho_{1,8}^{\bar{B}}=\left(m_{c} / m_{b}\right) \rho_{1,8}^{D}$, the values $\rho_{1}^{\bar{B}}=0.4$ and $\rho_{8}^{\bar{B}}=0.8$ are obtained for $m_{\bar{q}}=0.3 \mathrm{GeV}$. These values are relatively badly known, and are the leading source of uncertainty in our theoretical estimates. Since $\left|V_{q s}\right|^{2} /\left|V_{q d}\right|^{2} \approx 20,0.05$ for $q=u, c$, one can see from eq. (2.6) that the $t \rightarrow \bar{B}^{0}+u, \bar{B}_{s}^{0}+c$ decays are enhanced by a factor of $\times 20$ compared to the $t \rightarrow \bar{B}^{0}+c, \bar{B}_{s}^{0}+u$ ones, respectively.

The final numerical result for the partial width of a top quark into $\bar{B}^{0}+u\left(\right.$ or $\left.\bar{B}_{s}^{0}+c\right)$ in the NRQCD model is $5.4 \cdot 10^{-5} \mathrm{GeV}$, which for $\Gamma_{t}=1.35 \mathrm{GeV}[1]$ results into the corresponding top-quark branching ratios of $\mathcal{B}\left(t \rightarrow \bar{B}^{0}+u, \bar{B}_{s}^{0}+c\right)=\Gamma\left(t \rightarrow \bar{B}_{(s)}^{0}+u(c)\right) / \Gamma_{\mathrm{t}}=4 \cdot 10^{-5}$ and $\mathcal{B}\left(t \rightarrow \bar{B}^{0}+c, \bar{B}_{s}^{0}+u\right)=2.1 \cdot 10^{-6}$. We assign to those numerical results theoretical uncertainties of the order of $100 \%$, dominated by the non-perturbative transition probabilities $\rho_{1,8}^{\bar{B}}$. The sum of all such two-body decay modes has a total branching fraction of $\mathcal{B}\left(t \rightarrow \bar{B}_{(s)}^{0}+\right.$ jet $)=8.4 \cdot 10^{-5}$. The first result of our study is, therefore, that top quarks have a small but non-negligible (1-in-12000) probability to decay into the 2-body states, $t \rightarrow \bar{B}^{0}+u, c$ and $t \rightarrow \bar{B}_{s}^{0}+c, u$, plotted in the left and center panels of figure 1. All individual partial decay widths and branching fractions computed for each one of the four channels are listed in table 2 .

\subsection{Top decays into an $\Upsilon(n S)$-meson plus a $u$-or $c$-quark}

Following the theoretical approach described above, we can similarly calculate the CKMsuppressed $t \rightarrow \Upsilon(n S)+c, u$ decay (figure 1 right). In this process, the NRQCD velocity scaling rule is well applicable since the relative velocity between the two bottom quarks is $v \simeq \sqrt{0.1}$. We note first that the $t \rightarrow \Upsilon(n S)+u$ decay is suppressed by a factor of $\left|V_{u b}\right|^{2} /\left|V_{c b}\right|^{2} \approx 10^{-2}$ compared to the $t \rightarrow \Upsilon(n S)+c$ one, and therefore we will just 


\begin{tabular}{|lcc|}
\hline Decay mode & Partial decay width $(\mathrm{GeV})$ & Branching fraction \\
\hline$t \rightarrow \bar{B}^{0}+u$ & $5.4 \cdot 10^{-5}$ & $4.0 \cdot 10^{-5}$ \\
$t \rightarrow \bar{B}^{0}+c$ & $2.9 \cdot 10^{-6}$ & $2.1 \cdot 10^{-6}$ \\
\hline$t \rightarrow \bar{B}_{s}^{0}+c$ & $5.4 \cdot 10^{-5}$ & $4.0 \cdot 10^{-5}$ \\
$t \rightarrow \bar{B}_{s}^{0}+u$ & $2.9 \cdot 10^{-6}$ & $2.1 \cdot 10^{-6}$ \\
\hline$t \rightarrow \bar{B}_{(s)}^{0}+$ jet (total) & $1.14 \cdot 10^{-4}$ & $8.4 \cdot 10^{-5}$ \\
\hline
\end{tabular}

Table 2. Partial widths and branching fractions of top-quark two-body decays into a $\bar{B}_{(s)}^{0}$ meson plus a jet, computed here. The branching fractions assume a total top width of $\Gamma_{t}=1.35 \mathrm{GeV}$ [1]. (Theoretical uncertainties, of the order of $100 \%$, not quoted).

focus on the latter hereafter. The LO expressions for producing a bottomonium $H_{b \bar{b}}$ state, neglecting the subleading terms suppressed by factors of $m_{b}^{2} / m_{W}^{2}$ and/or $m_{q}^{2} / m_{b}^{2}$, are

$$
\begin{aligned}
& \Gamma\left(t \rightarrow\left(H_{b \bar{b}}\right)_{{ }_{3} S_{1}^{[1]}}+q\right)=\frac{\left\langle\mathcal{O}^{\left.H_{b \bar{b}}\left({ }^{3} S_{1}^{[1]}\right)\right\rangle}\right.}{m_{b}^{3}} \frac{\Gamma_{0}\left(t \rightarrow(b \bar{b})_{S}+q\right)}{216}, \\
& \Gamma\left(t \rightarrow\left(H_{b \bar{b}}\right)_{{ }_{3} S_{1}^{[8]}}+q\right)=\frac{\left\langle\mathcal{O}^{H_{b \bar{b}}}\left({ }^{3} S_{1}^{[8]}\right)\right\rangle}{m_{b}^{3}} \frac{\Gamma_{0}\left(t \rightarrow(b \bar{b})_{S}+q\right)}{36}, \\
& \Gamma\left(t \rightarrow\left(H_{b \bar{b}}\right)_{{ }^{1} S_{0}^{[8]}}+q\right)=\frac{\left\langle\mathcal{O}^{H_{b \bar{b}}}\left({ }^{1} S_{0}^{[8]}\right)\right\rangle}{m_{b}^{3}} \frac{\Gamma_{0}\left(t \rightarrow(b \bar{b})_{S}+q\right)}{12},
\end{aligned}
$$

for the $S$-wave states, and

$$
\begin{aligned}
& \Gamma\left(t \rightarrow\left(H_{b \bar{b}}\right)_{3 P_{0}^{[8]}}+q\right)=\frac{\left\langle\mathcal{O}^{H_{b \bar{b}}}\left({ }^{3} P_{0}^{[8]}\right)\right\rangle}{m_{b}^{5}} \frac{\Gamma_{0}\left(t \rightarrow(b \bar{b})_{P}+q\right)}{36}, \\
& \Gamma\left(t \rightarrow\left(H_{b \bar{b}}\right)_{3} P_{1}^{[8]}+q\right)=\frac{\left\langle\mathcal{O}^{H_{b \bar{b}}}\left({ }^{3} P_{1}^{[8]}\right)\right\rangle}{m_{b}^{5}} \frac{\Gamma_{0}\left(t \rightarrow(b \bar{b})_{P}+q\right)}{18}\left(1-2 \frac{m_{W}^{2}}{m_{t}^{2}}\right)^{2}, \\
& \Gamma\left(t \rightarrow\left(H_{b \bar{b}}\right)_{3} P_{2}^{[8]}+q\right)=\frac{\left\langle\mathcal{O}^{H_{b \bar{b}}\left({ }^{3} P_{2}^{[8]}\right)}\right.}{m_{b}^{5}} \frac{\Gamma_{0}\left(t \rightarrow(b \bar{b})_{P}+q\right)}{90}, \\
& \Gamma\left(t \rightarrow\left(H_{b \bar{b}}\right)_{{ }_{3} P_{0}^{[1]}}+q\right)=\frac{\left\langle\mathcal{O}^{\left.H_{b \bar{b}}\left({ }^{3} P_{0}^{[1]}\right)\right\rangle}\right.}{m_{b}^{5}} \frac{\Gamma_{0}\left(t \rightarrow(b \bar{b})_{P}+q\right)}{216}, \\
& \Gamma\left(t \rightarrow\left(H_{b \bar{b}}\right)_{{ }^{3} P_{1}^{[1]}}+q\right)=\frac{\left\langle\mathcal{O}^{H_{b \bar{b}}}\left({ }^{3} P_{1}^{[1]}\right)\right\rangle}{m_{b}^{5}} \frac{\Gamma_{0}\left(t \rightarrow(b \bar{b})_{P}+q\right)}{108}\left(1-2 \frac{m_{W}^{2}}{m_{t}^{2}}\right)^{2}, \\
& \Gamma\left(t \rightarrow\left(H_{b \bar{b}}\right)_{{ }_{3} P_{2}^{[1]}}+q\right)=\frac{\left\langle\mathcal{O}^{\left.H_{b \bar{b}}\left(3 P_{1}^{[2]}\right)\right\rangle}\right.}{m_{b}^{5}} \frac{\Gamma_{0}\left(t \rightarrow(b \bar{b})_{P}+q\right)}{540},
\end{aligned}
$$




\begin{tabular}{|ccccc|}
\hline top decay & \multicolumn{5}{c|}{ Partial decay width $\left(10^{-9} \mathrm{GeV}\right)$} \\
(bottomonium state) & Set I & Set II & Set III & Set IV \\
\hline$t \rightarrow \Upsilon(1 S)+q$ & 1.62 & 2.01 & 1.61 & 1.36 \\
$t \rightarrow \Upsilon(2 S)+q$ & 0.71 & 0.23 & 0.67 & 0.57 \\
$t \rightarrow \Upsilon(3 S)+q$ & 0.51 & 0.47 & 0.42 & 0.37 \\
\hline$t \rightarrow \Upsilon(n S)+q$ & 2.84 & 2.71 & 2.70 & 2.30 \\
\hline
\end{tabular}

Table 3. Partial widths (in units of $10^{-9} \mathrm{GeV}$ ) of inclusive top-quark decays into $\Upsilon(1 S, 2 S, 3 S$ ) mesons (including feed-down contributions from higher-excited bottomonia decays) plus a quark, for the four LDME sets considered in this work.

for the $P$-wave states, where $\left\langle\mathcal{O}^{H} b \bar{b}\left({ }^{2 s+1} L_{j}^{[c]}\right)\right\rangle$ are the LDMEs encoding the nonperturbative formation of the $\Upsilon(n S)$ bound state, and we define

$$
\begin{aligned}
& \Gamma_{0}\left(t \rightarrow(b \bar{b})_{S}+q\right) \equiv\left|V_{q b}\right|^{2} \frac{\alpha^{2} \pi m_{t}^{3} m_{b}^{2}}{\left(m_{t}^{2}-2 m_{W}^{2}\right)^{2} \sin ^{4} \theta_{w}}, \\
& \Gamma_{0}\left(t \rightarrow(b \bar{b})_{P}+q\right) \equiv\left|V_{q b}\right|^{2} \frac{\alpha^{2} \pi m_{t}^{7} m_{b}^{2}}{\left(m_{t}^{2}-2 m_{W}^{2}\right)^{4} \sin ^{4} \theta_{w}} .
\end{aligned}
$$

For $\Upsilon$ mesons, the NRQCD velocity scaling rule suggests that $P$-wave states have the same power counting as the colour-octet $S$-wave channels, which in any case are much smaller than the leading-power counting channel driven by the colour-singlet $S$-wave, ${ }^{3} S_{1}^{[1]}$. We include here all orbital channels because their associated LDMEs are well known (which is clearly not the case for the $\bar{B}^{0}$ mesons considered in the previous section). In our numerical results, we keep all the $m_{b}$ and $m_{c}$ dependencies at LO with the parameters listed in table 1 . In addition, similar to what has been done in ref. [11], we consider four different sets of non-perturbative LDMEs for bottomonia production:

- Set I: this set is based on the LDMEs presented in ref. [12], with the $\chi_{b}(3 P)$ ones set to zero.

- Set II: this set is based on the bottomonia LDMEs extracted in ref. [13], where the $\chi_{b}(3 P)$ contributions are also ignored.

- Set III: unlike the previous two sets, we used the LDMEs extracted from next-toleading-order (NLO) analyses. The LDMEs of $\Upsilon(n S), \chi_{b}(n P), 1 \leq n \leq 3$ are from ref. [14].

- Set IV: a second set of LDMEs based on NLO calculations taken from ref. [15] for bottomonia.

The final numerical values of the partial widths for the various bottomonium state decays, after including all feed-down contributions, are listed in table 3 . 


\begin{tabular}{|lcc|}
\hline Decay mode & Partial decay width $(\mathrm{GeV})$ & Branching fraction \\
\hline$t \rightarrow \Upsilon(n S)+c$ & $2.5 \cdot 10^{-9}$ & $1.9 \cdot 10^{-9}$ \\
$t \rightarrow \Upsilon(n S)+u$ & $2.5 \cdot 10^{-11}$ & $1.9 \cdot 10^{-11}$ \\
\hline
\end{tabular}

Table 4. Partial widths and branching fractions of top-quark two-body decays into an $\Upsilon$ meson plus a jet computed here. The branching fractions consider a total top-quark width of $\Gamma_{t}=1.35 \mathrm{GeV}[1]$. (Theoretical uncertainties, of the order of $10 \%$, not quoted).

The $\Upsilon(1 S, 2 S, 3 S)+$ quark decays add up to a partial width around $2.5 \cdot 10^{-9} \mathrm{GeV}$ (with a small $\sim 10 \%$ theoretical uncertainty given by the span of the results obtained with the different LDME sets listed in table 3), which is four orders of magnitude smaller than that corresponding to the $\bar{B}_{(s)}^{0}$ mode in the recombination mechanism (table 2). Such a large difference is explained by the very small probability of the $W$ boson to decay into bottom quarks to start with, $\left|V_{c b, u b}\right|^{2} /\left|V_{u d, c s}\right|^{2} \approx 10^{-3}-10^{-5}$, followed by the suppression of $\Upsilon$ colour-octet channels. The individual partial widths and branching fractions computed for the top decays into bottomonium+jet are listed in table 4 .

\section{Measurement of $t \rightarrow \bar{B}_{(s)}^{0}+$ jet in pp collisions at the LHC and FCC}

From the computed branching fractions of the rare two-body top decays listed in tables 2 and 4 , it is clear that the possibility to experimentally observe any of them is small and should be focused solely on the $\bar{B}_{(s)}^{0}$-meson-plus-jet final states, as they are at least four orders-of-magnitude more probable than the $\Upsilon$-plus-jet ones. In order to maximize the size of the top-quark data sample, we consider top-quark pair $(t \bar{t})$ production in pp collisions at $\sqrt{s}=14 \mathrm{TeV}$ during the HL-LHC phase, as well as at $\sqrt{s}=100 \mathrm{TeV}$ at the FCC. The rare two-body decays under consideration here have branching ratios, $\mathcal{O}\left(10^{-5}\right)$, of the same order as (or two orders-of-magnitude larger than) those accessible in BSM searches for rare $t \rightarrow q \gamma, q Z$ decays via flavour-changing neutral currents at the HL-LHC [16] and FCC [5], which also exploit the very large top-quark data samples available at both machines.

In the conservative estimates presented hereafter, we will assume detector acceptances and reconstruction performances typical of the current LHC analyses. At the HL-LHC, the upgraded machine and detectors will function at luminosities $5-7.5 \cdot 10^{34} \mathrm{~cm}^{-2} \mathrm{~s}^{-1}$, corresponding to an average pileup of 140-200 pp collisions per bunch crossing. Advanced pileup mitigation techniques have been therefore designed to keep the top-quark reconstruction under control [6], and no significant degradation of the energy scale and resolution of the jets, the missing transverse energy (neutrinos), nor the $b$-jet identification are expected compared to the current ATLAS and CMS performances. ${ }^{2}$ Any unexpected loss in the top-quark reconstruction efficiency due to potentially increased kinematic thresholds (at the trigger or offline analysis levels) will be compensated by the higher $t \bar{t}$ cross section at $14 \mathrm{TeV}$ compared to the $13-\mathrm{TeV}$ operation so far, as well as by the increased (forward)

\footnotetext{
${ }^{2}$ The LHCb detector has unparalleled $\bar{B}_{(s)}^{0}$ reconstructions capabilities [17-32], albeit with (smaller) forward-only acceptance compared to ATLAS/CMS. Although $t \bar{t}$ production has been already observed in its dilepton final state [33, 34], LHCb will integrate about a factor of 10 lower luminosities than ATLAS and CMS in the HL-LHC phase, and the feasibility of this search would thereby require a dedicated analysis beyond this first exploratory study.
} 
acceptance and higher granularity of the upgraded detectors, compared to the LHC conditions today. At the FCC, the experiments aim at fully tracking coverage over a large pseudorapidity $|\eta|<5$ region, leading to $\sim 1.7$ times effectively larger acceptance for topquark detection than currently at the LHC, with a detector granularity also adapted to cope with the expected $\mathcal{O}(200-1000)$ pileup collisions.

\subsection{Expected top-quark yields}

The total pp $\rightarrow t \bar{t}+X$ cross sections at $\sqrt{s}=14$ and $100 \mathrm{TeV}$ are computed with Top ++ v2.0 [35] at next-to-next-to-leading order (NNLO) plus next-to-next-to-leading-log (NNLL) accuracies, using the NNLO NNPDF3.0 parton distribution functions (PDF) [36]. The code is run with $\mathrm{N}_{\mathrm{f}}=5$ flavours, top pole masses set to $m_{t}=173.0 \mathrm{GeV}$, default renormalization and factorization scales set to $\mu_{\mathrm{R}}=\mu_{\mathrm{F}}=m_{t}$, and QCD coupling set to $\alpha_{\mathrm{s}}=0.1180$. Such a theoretical NNLO+NNLL setup yields theoretical cross sections that are in very good agreement with all the experimental data measured so far in pp collisions at $\sqrt{s}=7,8,13 \mathrm{TeV}$ at the LHC [1]. The computed NNLO+NNLL cross sections amount to $\sigma_{t \bar{t}}=980 \pm 17(\mathrm{PDF})_{-35}^{+24}$ (scale) pb at the LHC, and $\sigma_{t \bar{t}}=34.80 \pm 1.20(\mathrm{PDF})_{-1.65}^{+1.00}$ (scale) nb at the FCC. The PDF uncertainties, of the order of $\pm 1.8 \%$ at the LHC and $\pm 3.5 \%$ at the FCC, are obtained from the 100 replicas of the NNPDF3.0 set. The theoretical scale uncertainty, amounting to $+2.5 \%,-3.5 \%$ at the $\mathrm{LHC}$ and $+2.9 \%,-4.7 \%$ at the FCC, is estimated by modifying $\mu_{\mathrm{R}}$ and $\mu_{\mathrm{F}}$ within a factor of two with respect to their default value. The computed total cross sections are listed in the first column of table 5. From these cross sections, in the narrow-width approximation $\Gamma_{t} \ll m_{t}$ where production and decay processes factorize, one would expect a total inclusive number of two-body-decay events of the order of:

$$
\begin{aligned}
N_{\mathrm{t} \rightarrow \overline{\mathrm{B}}_{(\mathrm{s})}^{0}+\mathrm{j}}^{t \bar{t} \text {,vts }} & =\sigma_{t \bar{t}} \times \mathcal{L}_{\text {int }} \times \mathcal{B}\left(t \rightarrow \bar{B}_{(s)}^{0}+\text { jet }\right) \approx \\
& \approx 1 \mathrm{nb} \times 3 \cdot 10^{9} \mathrm{nb}^{-1} \times 8.4 \cdot 10^{-5} \approx 2.5 \cdot 10^{5}
\end{aligned}
$$

at the HL-LHC, and

$$
N_{\mathrm{t} \rightarrow \overline{\mathrm{B}}_{(\mathrm{s})}^{0}+\mathrm{j}}^{t \bar{t}, \mathrm{evts}} \approx 35 \mathrm{nb} \times 2 \cdot 10^{10} \mathrm{nb}^{-1} \times 8.4 \cdot 10^{-5} \approx 6 \cdot 10^{7}
$$

at the FCC. The numbers of rare top-quark decays at the FCC is 250 times larger than at the LHC, thanks to $\times 35$ and $\times 7$ larger cross sections and integrated luminosities, respectively

Of course, the number of such decays actually visible will be smaller after taking into account detector acceptance and reconstruction efficiency losses for the final states under consideration. Depending on the decay of one or both $W$ bosons, the final states for the top-pair process can be divided into three classes [1]:

- Fully hadronic: $t \bar{t} \rightarrow W^{+} b W^{-} \bar{b} \rightarrow q^{\prime} \bar{q} b \bar{q}^{\prime \prime} q^{\prime \prime \prime} \bar{b}(\mathcal{B}=45.7 \%)$

- Lepton + jets: ${ }^{3} t \bar{t} \rightarrow W^{+} b W^{-} \bar{b} \rightarrow q^{\prime} \bar{q} b \ell^{-} \nu_{\ell} \bar{b}(\mathcal{B}=43.8 \%)$

- Dileptons: $t \bar{t} \rightarrow W^{+} b W^{-} \bar{b} \rightarrow \ell^{+} \nu_{\ell} b \ell^{-} \nu_{\ell} \bar{b}(\mathcal{B}=10.5 \%)$

\footnotetext{
${ }^{3}$ This decay $\mathcal{B}$ includes both charge-conjugate modes.
} 


\begin{tabular}{|lc|cc|}
\hline$\sqrt{s}$ & $\sigma_{\mathrm{NNLO}+\mathrm{NNLL}}^{t \bar{t} \text {,incl. }}$ & \multicolumn{2}{|c|}{ top-quark pair $(\mathcal{B} \times$ acceptance $\times$ efficiency $)$ losses } \\
& & fully hadronic & $\ell+$ jets \\
\hline $14 \mathrm{TeV}$ & $980 \pm 17(\mathrm{PDF})_{-35}^{+24}(\mathrm{scale}) \mathrm{pb}$ & $0.457 \times 0.57 \times 0.75=0.20$ & $0.35 \times 0.64 \times 0.75=0.17$ \\
$100 \mathrm{TeV}$ & $34.80 \pm 1.20(\mathrm{PDF})_{-1.65}^{+1.00}($ scale $) \mathrm{nb}$ & $0.457 \times 0.76 \times 0.75=0.26$ & $0.35 \times 0.71 \times 0.75=0.19$ \\
\hline
\end{tabular}

Table 5. Top-pair cross sections computed at NNLO+NNLL accuracy with Top++ v2.0, and product of branching ratios $\times$ acceptance $\times$ efficiency losses for the fully-hadronic and $\ell+$ jets (with $\ell^{ \pm}=e^{ \pm}, \mu^{ \pm}$) final states (for the selection criteria listed in sections 3.1 .1 and 3.1 .2 respectively, computed with MADGraph5_AMC@NLO), in pp collisions at $\sqrt{s}=14$ and $100 \mathrm{TeV}$.

Obviously, the last purely leptonic final states cannot be used for searches of the rare 2body hadronic decays considered here, which leaves us with $90 \%$ of the $t \bar{t}$ cross section, with fully-hadronic and lepton+jets final states, to be exploited as described next.

\subsubsection{Fully hadronic $t \bar{t}$ final state}

One can first attempt the $t \rightarrow \bar{B}_{(s)}^{0}+q$ measurement in the multi-quark decay of a $t \bar{t}$ pair: $t \bar{t} \rightarrow W^{+} b W^{-} \bar{b} \rightarrow q\left(\bar{q}^{\prime} b\right) q^{\prime \prime} \bar{q}^{\prime \prime \prime} \bar{b}$, where both $W$ bosons decay hadronically (and, in our case of interest, one of its down-type quarks recombines with the closest $b$-quark to form a neutral $B$ meson). Such a fully-hadronic $t \bar{t}$ final state has the largest branching fraction $(\mathcal{B}=0.457)$, and provides two top quarks per event, although it has the experimental drawback of a large combinatorial background from QCD multi-jets processes. Notwithstanding this difficulty, ATLAS and CMS have managed to setup online triggers that can collect all hadronic $t \bar{t}$ events without any level-1 prescale rate. Typical selection criteria used by the ATLAS/CMS experiments [37-42] are:

- At least 4 jets (reconstructed with the anti- $k_{\mathrm{T}}$ algorithm $[43,44]$ with distance parameter $R=0.5)$ with $p_{\mathrm{T}}>25 \mathrm{GeV}$ and $|\eta|<3.0(|\eta|<5.0$ at the FCC);

- A total scalar sum of transverse energy in the reconstructed jets above roughly twice the top mass, $H_{\mathrm{T}}>350 \mathrm{GeV}$;

- At least $1 b$ jet tagged with a typical $75 \%$ efficiency;

Based on such an event signature, the HL-LHC studies indicate that unprescaled triggers can collect all relevant $t \bar{t}$ events at level-1 without any significant loss [6] although, if needed, one can further require at higher-level trigger (HLT) the extra presence of a high- $p_{\mathrm{T}} \bar{B}^{0}$ meson, to reduce the collection rate of potentially large backgrounds. We generate $t \bar{t}$ events with MADGraPh5_AMC@NLO v2.6.6 (MG5_AMC henceforth) [45] using the NNPDF30_nlo_as_0118 PDF [36], implementing (at the parton level) the selection criteria listed above, and obtain yield losses from the combined acceptance $\times$ efficiency $=0.57 \times 0.75=0.43$ and $0.76 \times 0.75=0.57$ at the HL-LHC and FCC, respectively (table 5). Combining those values with the numbers (3.1)-(3.2) and the over- 
all branching ratio, ${ }^{4}$ results in $N_{\mathrm{t} \rightarrow \overline{\mathrm{B}}_{(\mathrm{s})}^{t \bar{l}}+\mathrm{j}}^{\text {full had. }}=2 \times 2.5 \cdot 10^{5} \times \sqrt{0.457} \times 0.43 \approx 1.5 \cdot 10^{5}$, and $2 \times 6 \cdot 10^{7} \times \sqrt{0.457} \times 0.57 \approx 4.5 \cdot 10^{7}$ top-quarks theoretically reconstructible in the (sum of) $t \rightarrow \bar{B}_{(s)}^{0}+q$ decay modes in fully-hadronic $t \bar{t}$ samples, at the HL-LHC and FCC, respectively.

\subsubsection{Lepton + jets $t \bar{t}$ final state}

The "cleanest" channel to attempt the two-body top-decay measurement is the lepton+jets one, $t \bar{t} \rightarrow W^{+} b W^{-} \bar{b} \rightarrow q\left(\bar{q}^{\prime} b\right) \ell \nu_{\ell} \bar{b}$, where $\ell$ stands for an electron or muon and, in our case of interest, one of the down-type quarks from the $W$ boson decay further recombines with the closest $b$-quark to form a neutral $B$ meson. As done commonly at the LHC, we exclude the channels with hadronically-decaying tau leptons, which are more difficult to reconstruct, but include electrons and muons from tau decays that have a combined top-quark branching fraction of $\mathcal{B}=6.4 \%$. Compared to the fully-hadronic mode, the $\ell$-plus-jets channel has a slightly smaller $\mathcal{B} \approx 35 \%$ overall branching ratio, and half the number of top quarks available for the 2-body decay, but it features a cleaner experimental signature based on the presence of a high $p_{\mathrm{T}}$ isolated electron or muon. The following selection criteria, typically used by the ATLAS/CMS experiments [46-51], are applied:

- One isolated (cone radius $R_{\text {isol }}=0.3$ ) charged lepton $\ell$ with $p_{\mathrm{T}}>30 \mathrm{GeV}$ and $|\eta|<$ $2.5(|\eta|<5.0$ at the FCC);

- At least 2 jets (reconstructed with the anti- $k_{\mathrm{T}}$ algorithm with $R=0.5$ ) with $p_{\mathrm{T}}>$ $25 \mathrm{GeV}$ and $|\eta|<3.0(|\eta|<5.0$ at the FCC), and separated from the lepton by $\Delta R(\ell, j)>0.4$

- $1 b$-jet tagged with a typical $75 \%$ efficiency;

Based on such an event signature (one isolated high- $p_{\mathrm{T}}$ charged lepton, plus at least 2 jets of which one is $b$-tagged), a trigger can be implemented to collect all relevant $t \bar{t}$ events unprescaled without any significant loss. [If needed to reduce any unforeseen extra background, one could also add as HLT requirements a minimum missing transverse energy from the unobserved $\nu$, and/or the extra presence of a high- $p_{\mathrm{T}} \bar{B}^{0}$ meson in the event.] The impact of such selection criteria, evaluated again at NLO accuracy with MG5_AMC at the parton level, indicates a $64 \%(71 \%)$ acceptance of the $\ell+$ jets $t \bar{t}$ cross section at $14(100) \mathrm{TeV}$ (table 5). These numbers are very similar to those obtained with the MCFM v8.0 code [52], with a very small dependence on the underlying PDF [53]. The extra requirement to have one $b$-tagged jet in the event results in a final combined acceptance $\times$ efficiency $\approx 48 \%(53 \%)$ to collect such a $t \bar{t}$-enriched sample at 14 (100) TeV. Thus, combining the numbers (3.1)(3.2) with the overall $\ell+$ jets branching ratio and the acceptance and efficiency losses, we expect about $N_{\mathrm{t} \rightarrow \overline{\mathrm{B}}_{(\mathrm{s})}^{\mathrm{t}} \mathrm{\ell}+\mathrm{j}}^{\mathrm{j}}=2.5 \cdot 10^{5} \times 0.35 / \sqrt{0.457} \times 0.48 \approx 6.2 \cdot 10^{4}$ and $6 \cdot 10^{7} \times 0.35 / \sqrt{0.457} \times$

\footnotetext{
${ }^{4}$ Note that we count two top quarks per event and that the fully hadronic $t \bar{t}$ branching fraction enters as a square-root, $\sqrt{0.457}$, given that one top decay to $\bar{B}_{(s)}^{0}+$ jet is already included in the $2.5 \cdot 10^{5}$ and $6 \cdot 10^{7}$ event counts.
} 
$0.53 \approx 1.6 \cdot 10^{7}$ top-quarks theoretically reconstructible in the (sum of) $t \rightarrow \bar{B}_{(s)}^{0}+q$ decay modes at the HL-LHC and FCC, respectively.

\subsection{Measurement of the $t \rightarrow \bar{B}_{(s)}^{0}+$ jet decay}

The last step needed to experimentally observe the rare top decays considered here relies on estimating the acceptance and efficiency to measure the decay $\bar{B}_{(s)}^{0}$ mesons, given that the reconstruction of the accompanying back-to-back $(u, c)$ jet is already accounted for in the event count after the typical selection criteria discussed in the previous section. For the full $\bar{B}_{(s)}^{0}$-meson reconstruction required in this work, one cannot use the $\bar{B}_{(s)}^{0} \rightarrow \ell \nu_{\ell} X$ (semi)leptonic decays with undetected neutrinos in the event, and must focus instead in the most abundant hadronic modes involving the $b \rightarrow c$ transition at the quark level either producing a charmonium- or charmed-meson plus lighter hadrons in the final state, $\bar{B}_{(s)}^{0} \rightarrow J / \psi h h($ table 6$)$ or $\bar{B}_{(s)}^{0} \rightarrow D h(h)$ (table 8) with $h=\pi, K$. It is important to note that, for our signal events, the presence of a truly high- $p_{\mathrm{T}} \bar{B}_{(s)}^{0}$-meson - carrying at least a momentum corresponding to half of the top-quark mass, $p_{\mathrm{T}}^{B} \gtrsim 85 \mathrm{GeV}$, i.e. with about a factor of 10 larger transverse momenta than those typically studied $\left(p_{\mathrm{T}}^{B} \gtrsim 5 \mathrm{GeV}\right)$ in more inclusive $B$-meson measurements at the LHC - will significantly boost all final decay hadrons $(J / \psi, D, \pi$, and $K)$, thereby improving the reconstruction performance. The detector acceptance for each final state can be realistically quantified as discussed below, although the full computation of $\mu / \pi / K$ reconstruction efficiencies would require a dedicated analysis of additional signal losses (due to tracking and secondary vertexing inefficiency, particle misidentification, combinatorial backgrounds, bin migrations, detector resolution effects, etc.) that go well beyond this first feasibility study. Since all LHC studies indicate that the reconstruction efficiency (in particular for tracking and vertexing) increases steadily with $p_{\mathrm{T}}^{B}$ for all decay modes [17-23, 23-32, 54-67], no extra reconstruction efficiency loss will be hereafter assumed in the estimation of final event yields.

\subsubsection{Final states with $J / \psi$ mesons}

The ATLAS [54-56], CMS [23, 58-65], and LHCb [17-24] experiments have measured neutral $B$ mesons in their clean exclusive decays into $J / \psi$, followed by its dimuon decay with $\mathcal{B}\left(J / \psi \rightarrow \mu^{+} \mu^{-}\right)=5.96 \%$, plus kaons and/or pions, with the branching ratios listed in table 6 . The combined branching fractions for such final states are rather low, in the $10^{-4}-10^{-5}$ range, but relatively free of backgrounds.

The typical reconstruction of the $\bar{B}_{(s)}^{0}$ events of interest proceeds as follows (see e.g. [64] for measurements of $B \rightarrow J / \psi+X$ decays in the $t \bar{t}$ lepton + jet final states of relevance here). The $\bar{B}_{(s)}^{0}$ candidates are obtained by: (i) reconstructing two muons within $|\eta|<3$ (5 at FCC) and matching their invariant mass to that of a non-prompt $J / \psi$, (ii) reconstructing two additional tracks over $|\eta|<3$ (5 at FCC) and requiring them (with charged pion and/or kaon mass hypothesis) to have an invariant mass consistent with any intermediate hadronic resonance $\left(K^{*}(892)^{0}, K_{s}^{0}, \phi(1020), \ldots\right)$ if present, (iii) fitting the charged tracks to a common secondary vertex consistent with the $\bar{B}_{(s)}^{0}$-meson lifetime, and finally (iv) requiring the $J / \psi$ and accompanying (resonant or not) hadronic state to have an invariant mass 


\begin{tabular}{|llll|}
\hline $\bar{B}_{(s)}^{0}$ meson & Total branching fraction & \multicolumn{2}{c|}{ Acceptance } \\
decay mode & (product of individual $\mathcal{B}$ 's $)$ & LHC & FCC \\
\hline $\bar{B}^{0} \rightarrow J / \psi\left(\mu^{+} \mu^{-}\right) K^{* 0}\left(K^{ \pm} \pi^{\mp}\right)$ & $1.27 \cdot 10^{-3} \times 0.0596 \times 1 \approx 7.6 \cdot 10^{-5}$ & $50 \%$ & $55 \%$ \\
$\bar{B}^{0} \rightarrow J / \psi\left(\mu^{+} \mu^{-}\right) K^{+} \pi^{-}$ & $1.15 \cdot 10^{-3} \times 0.0596 \approx 6.9 \cdot 10^{-5}$ & $60 \%$ & $70 \%$ \\
$\bar{B}^{0} \rightarrow J / \psi\left(\mu^{+} \mu^{-}\right) K_{s}^{0}\left(\pi^{+} \pi^{-}\right)$ & $0.87 \cdot 10^{-3} \times 0.0596 \times 0.69 \approx 3.6 \cdot 10^{-5}$ & $50 \%$ & $60 \%$ \\
\hline $\bar{B}_{s}^{0} \rightarrow J / \psi\left(\mu^{+} \mu^{+}\right) \phi\left(K^{+} K^{-}\right)$ & $1.08 \cdot 10^{-3} \times 0.0596 \times 0.49 \approx 3.2 \cdot 10^{-5}$ & $60 \%$ & $65 \%$ \\
$\bar{B}_{s}^{0} \rightarrow J / \psi\left(\mu^{+} \mu^{+}\right) K^{+} K^{-}$ & $0.79 \cdot 10^{-3} \times 0.0596 \approx 4.7 \cdot 10^{-5}$ & $65 \%$ & $70 \%$ \\
$\bar{B}_{s}^{0} \rightarrow J / \psi\left(\mu^{+} \mu^{+}\right) \pi^{+} \pi^{-}$ & $0.21 \cdot 10^{-3} \times 0.0596 \approx 1.25 \cdot 10^{-5}$ & $65 \%$ & $70 \%$ \\
\hline
\end{tabular}

Table 6. Most important exclusive $\bar{B}^{0}$ and $\bar{B}_{s}^{0}$ decay modes into $J / \psi+$ hadrons, with total branching ratios (product of each consecutive decay, from [1]), and typical acceptances for the corresponding final-state decay products in the considered $(\mu, \pi, K)$ central detector acceptances at the LHC and FCC (see text).

matching the $\bar{B}_{(s)}^{0}$ one. In order to assess the geometrical acceptance of all decay channels of table 6 , we generate $t \bar{t}$ events with PYTHIA 8 v2.26 (PYTHIA 8, henceforth) [68], selecting only those where top quarks decay into a $\bar{B}_{(s)}^{0}$ meson followed by the decay of the latter into each individual mode listed, switched-on one by one in the code. We then verify whether the two muons from the $J / \psi$ and the pions and/or kaons are all within the detector acceptance (we take $|\eta|<3,5$ for all tracks at the LHC and FCC, respectively, and require both muons to have $p_{\mathrm{T}}>3 \mathrm{GeV}$ ). With such a Monte Carlo event generation setup, we obtain the acceptances listed in the last two columns of table 6 , which lie in the $50 \%$ to $70 \%$ range.

Based on the analysis outlined above, table 7 lists the final expected number of twobody $t \rightarrow \bar{B}_{(s)}^{0}+$ jet events in $t \bar{t}$ (fully hadronic, and $\ell+$ jets) final states with the $\bar{B}_{(s)}^{0}$-meson decaying into $J / \psi$ plus light hadrons, after accounting for branching ratios and detector acceptance losses (table 6) in pp collisions at the LHC and FCC. Among the $\bar{B}_{(s)}^{0}$-meson decay channels, the most promising ones in terms of final yields are $\bar{B}^{0} \rightarrow J / \psi K^{+} \pi^{-}$, $\bar{B}^{0} \rightarrow J / \psi K^{* 0}$, and $\bar{B}_{s}^{0} \rightarrow J / \psi K^{+} K^{-}$. We expect a total of about 16 and 5200 rare two-body top-quark signal events reconstructed at the LHC and FCC, respectively, with the final states with reconstructed $\bar{B}^{0}$ mesons being about twice more abundant than those with $\bar{B}_{s}^{0}$ mesons. The $t \bar{t}$ event selection based on fully-hadronic final states leads to about a factor of two larger signal counts than that from $\ell+$ jets. The final states with $c$ - or $u$-quark jets, accompanying the $\bar{B}_{(s)}^{0}$ meson, share evenly the final number of signal events. The total of $\sim 16$ signal events expected at the LHC indicate a difficult measurement of the 2-body top-quark decays, via an invariant mass analysis of $\bar{B}^{0}(J / \psi h h)$ channels plus a jet, and one will need a future hadron collider such as the FCC to carry out the measurement with about $\times 300$ times more reconstructed events available.

\subsubsection{Final states with $D^{0, \pm}$ mesons}

The previous section considered the measurement of two-body $\bar{B}_{(s)}^{0}+$ jet top-quark decays via $\bar{B}_{(s)}^{0}$ mesons reconstructed in their $J / \psi\left(\mu^{+} \mu^{-}\right) h h$ final states that feature very 


\begin{tabular}{|llcc|}
\hline Top pair & $\bar{B}_{(s)}^{0}$ meson & \multicolumn{2}{c|}{ Events after all selection cuts } \\
final state & decay mode & LHC $\left(3 \mathrm{ab}^{-1}\right)$ & FCC $\left(20 \mathrm{ab}^{-1}\right)$ \\
\hline$t \bar{t} \rightarrow \bar{B}^{0}+u, c$ & & \\
$\circ$ fully hadronic & $\bar{B}^{0} \rightarrow J / \psi\left(\mu^{+} \mu^{-}\right) K^{+} \pi^{-}$ & 3.1 & 1050 \\
$\circ \ell+$ jets & 1.4 & 380 \\
$\circ$ fully hadronic & $\bar{B}^{0} \rightarrow J / \psi\left(\mu^{+} \mu^{-}\right) K^{* 0}\left(K^{ \pm} \pi^{\mp}\right)$ & 2.7 & 940 \\
$\circ \ell+$ jets & & 1.2 & 340 \\
$\circ$ fully hadronic & $\bar{B}^{0} \rightarrow J / \psi\left(\mu^{+} \mu^{-}\right) K_{s}^{0}\left(\pi^{+} \pi^{-}\right)$ & 1.3 & 470 \\
$\circ \ell+$ jets & & 0.6 & 170 \\
Sum of all channels & & 10.2 & 3300 \\
\hline$t \bar{t} \rightarrow \bar{B}_{s}^{0}+c, u$ & & & 710 \\
$\circ$ fully hadronic & $\bar{B}_{s}^{0} \rightarrow J / \psi\left(\mu^{+} \mu^{+}\right) K^{+} K^{-}$ & 2.1 & 250 \\
$\circ \ell+$ jets & & 0.9 & 460 \\
$\circ$ fully hadronic & $\bar{B}_{s}^{0} \rightarrow J / \psi\left(\mu^{+} \mu^{+}\right) \phi\left(K^{+} K^{-}\right)$ & 1.4 & 170 \\
$\circ \ell+$ jets & & 0.6 & 200 \\
$\circ$ fully hadronic & $\bar{B}_{s}^{0} \rightarrow J / \psi\left(\mu^{+} \mu^{+}\right) \pi^{+} \pi^{-}$ & 0.6 & 70 \\
$\circ \ell+$ jets & & 0.3 & 1900 \\
Sum of all channels & & 5.7 & 5200 \\
\hline TOTAL & & 16 & \\
\hline
\end{tabular}

Table 7. Expected number of $t \rightarrow \bar{B}_{(s)}^{0}+$ jet events in selected $t \bar{t}$ final-states (fully hadronic, and $\ell+$ jets) with $\bar{B}^{0}$-mesons decaying into $J / \psi$ plus light hadrons, after estimating branching ratios and detector acceptance losses, for pp collisions at the LHC and FCC.

clean topologies albeit relatively small decay rates. Alternative $\bar{B}_{(s)}^{0}$ meson decays exist into multi- $\pi, K$ final states through an intermediate $D$ meson with larger branching ratios (for updated comprehensive $\bar{B}_{(s)}^{0}$ decay lists, see e.g. refs. [1, 69]). Potentially large combinatorial backgrounds for such channels can be reduced if one avoids final states with neutral hadrons and with more than four tracks, which can be more difficult to reconstruct under high pp pileup conditions. Namely, focusing on $\bar{B}_{(s)}^{0} \rightarrow D_{(s)}^{0, \pm} h(h)$ modes (with $h=\pi, K$ ) with $\mathcal{B} \approx 10^{-4}-10^{-3}$ followed by $\mathcal{B}\left(D^{+} \rightarrow K^{-} \pi^{+} \pi^{+}\right)=0.094$, $\mathcal{B}\left(D_{s}^{+} \rightarrow K^{-} K^{+} \pi^{+}\right)=0.055$, or $\mathcal{B}\left(D^{0} \rightarrow K^{-} \pi^{+}\right)=0.039$, with factors of $2-5$ larger final branching fractions than the $J / \psi$-based ones discussed in the previous subsection. Among those, the decays listed in table 8 feature the largest total branching ratios.

All experiments at the LHC (ATLAS [57], CMS [64, 66, 67], and LHCb [25-32]) have reconstructed ${ }^{5}$ the different $\bar{B}^{0} \rightarrow D \rightarrow 3 \pi K, 2 \pi 2 K$ decay chains listed in table 8 . Such

\footnotetext{
${ }^{5}$ In particular, ref. [64] has explicitly measured $B \rightarrow D$ meson decays in the $t \bar{t}$ lepton + jet final states of relevance here.
} 


\begin{tabular}{|llll|}
\hline $\bar{B}_{(s)}^{0}$ meson & Total branching fraction & \multicolumn{2}{c|}{ Acceptance } \\
decay mode & (product of individual $\mathcal{B}$ 's $)$ & LHC & FCC \\
\hline $\bar{B}^{0} \rightarrow D^{+}\left(K^{-} \pi^{+} \pi^{+}\right) \pi^{-}$ & $2.52 \cdot 10^{-3} \times 0.094 \approx 2.37 \cdot 10^{-4}$ & $55 \%$ & $65 \%$ \\
$\bar{B}^{0} \rightarrow \bar{D}^{*,+}\left(D^{0}\left(K^{-} \pi^{+}\right) \pi^{+}\right) \pi^{-}$ & $2.74 \cdot 10^{-3} \times 0.677 \times 0.039 \approx 7.2 \cdot 10^{-5}$ & $55 \%$ & $65 \%$ \\
$\bar{B}^{0} \rightarrow \bar{D}^{0}\left(K^{-} \pi^{+}\right) \pi^{+} \pi^{-}$ & $0.88 \cdot 10^{-3} \times 0.039 \approx 3.43 \cdot 10^{-5}$ & $75 \%$ & $80 \%$ \\
$\bar{B}^{0} \rightarrow D^{+}\left(K^{-} \pi^{+} \pi^{+}\right) K^{-}$ & $0.186 \cdot 10^{-3} \times 0.094 \approx 1.75 \cdot 10^{-5}$ & $55 \%$ & $65 \%$ \\
\hline $\bar{B}_{s}^{0} \rightarrow D_{s}^{+}\left(K^{-} K^{+} \pi^{+}\right) \pi^{-}$ & $3.0 \cdot 10^{-3} \times 0.055 \approx 1.6 \cdot 10^{-4}$ & $60 \%$ & $60 \%$ \\
$\bar{B}_{s}^{0} \rightarrow \bar{D}^{0}\left(K^{-} \pi^{+}\right) K^{-} \pi^{+}$ & $1.04 \cdot 10^{-3} \times 0.039 \approx 4 \cdot 10^{-5}$ & $60 \%$ & $65 \%$ \\
\hline
\end{tabular}

Table 8. Most important $\bar{B}^{0}$ and $\bar{B}_{s}^{0}$ decay modes into $D$-mesons plus one (or two) light mesons, with total branching ratios (product of each consecutive decay, from [1]), and typical acceptances for the corresponding final-state decay products in the considered $(\pi, K)$ central detector acceptances at the LHC and FCC.

analyses usually proceed as follows: (i) reconstructing two or three charged-hadron tracks within $|\eta|<3$ (5 at FCC) successfully fitted to a common vertex consistent with the $D$ meson lifetime, (ii) assigning to the tracks the pion or kaon masses, based on appropriate charge associations, and requiring the $m_{K \pi(\pi)}$ invariant mass to be consistent with the $D_{(s)}^{0, \pm}$ mass, and (iii) reconstructing one or two more charged-hadron tracks within $|\eta|<3$ (5 at FCC) that, combined with the $D_{(s)}^{0, \pm}$ candidate at a secondary vertex consistent with the $\bar{B}_{(s)}^{0}$-meson lifetime, have an invariant mass matching the $\bar{B}_{(s)}^{0}$ one. As done in the previous section, the geometric acceptance of all individual modes listed in table 8 is determined with $t \bar{t}$ events generated with PYTHIA 8, where the aforementioned analysis chain is implemented. Based on such a simulation setup, we obtain the acceptances listed in the last two columns of table 8 , which lie in the $55 \%$ to $80 \%$ range.

Following the method outlined above, table 9 lists the total expected number of twobody $t \rightarrow \bar{B}_{(s)}^{0}+$ jet events in $t \bar{t}$ (fully hadronic, and $\ell+$ jets) final states with $\bar{B}_{(s)}^{0}$-mesons decaying into $D^{0, \pm}$ plus light hadrons, after accounting for branching ratios and detector acceptance losses (table 8) for pp collisions at the LHC and FCC. As for the $J / \psi$ final states considered in the previous section, full reconstruction efficiency for the boosted final hadrons from high- $p_{\mathrm{T}} \bar{B}_{(s)}^{0}$ decays is assumed in the estimation of final event yields. Among the $\bar{B}_{(s)}^{0}$-meson decay channels, the most promising ones in terms of final yields are $\bar{B}^{0} \rightarrow$ $D^{+} \pi^{-}$, and $\bar{B}_{s}^{0} \rightarrow D_{s}^{+} \pi^{-}$. In total, we expect about 33 and 11000 rare two-body topquark signal events at the LHC and FCC, respectively, with about twice more final states reconstructed with $\bar{B}^{0}$ than $\bar{B}_{s}^{0}$ mesons. The final signal yields from the $t \bar{t}$ event selection based on fully-hadronic final states is about twice larger than that from $\ell+$ jets. Signal events are equally shared between those having a $c$ - or $u$-quark jet plus the $\bar{B}_{(s)}^{0}$ meson. With about 33 signal counts expected at the LHC, the observation of the 2-body top-quark decays via exclusive final states involving $\bar{B}_{(s)}^{0} \rightarrow D h(h)$ channels may be feasible, but such a measurement will definitely profit from the 11000 reconstructed events expected at the FCC. In the next section, we provide an alternative and simpler method to observe the rare 2-body top-quark decays with much larger data samples at both the LHC and FCC. 


\begin{tabular}{|llcc|}
\hline $\begin{array}{l}\text { Top pair } \\
\text { final state }\end{array}$ & $\bar{B}_{(s)}^{0}$ meson & \multicolumn{2}{c|}{ Events after all selection cuts } \\
\hline$t \bar{t} \rightarrow \bar{B}^{0}+u, c$ & LHC $\left(3 \mathrm{ab}^{-1}\right)$ & FCC $\left(20 \mathrm{ab}^{-1}\right)$ \\
$\circ$ fully hadronic & $\bar{B}^{0} \rightarrow D^{+}\left(K^{-} \pi^{+} \pi^{+}\right) \pi^{-}$ & 9.3 & 3500 \\
$\circ \ell+$ jets & 4 & 1200 \\
$\circ$ fully hadronic & $\bar{B}^{0} \rightarrow \bar{D}^{*,+}\left(D^{0}\left(K^{-} \pi^{+}\right) \pi^{+}\right) \pi^{-}$ & 2.8 & 1100 \\
$\circ \ell+$ jets & & 1.2 & 380 \\
$\circ$ fully hadronic & $\bar{B}^{0} \rightarrow \bar{D}^{0}\left(K^{-} \pi^{+}\right) \pi^{+} \pi^{-}$ & 1.8 & 630 \\
$\circ \ell+$ jets & & 0.8 & 220 \\
$\circ$ fully hadronic & $\bar{B}^{0} \rightarrow D^{+}\left(K^{-} \pi^{+} \pi^{+}\right) K^{-}$ & 0.7 & 250 \\
$\circ \ell+$ jets & & 0.3 & 90 \\
Sum of all channels & & 21.0 & 7300 \\
\hline$t \bar{t} \rightarrow \bar{B}_{s}^{0}+c, u$ & & & 2200 \\
$\circ$ fully hadronic & $\bar{B}_{s}^{0} \rightarrow D_{s}^{+}\left(K^{+} K^{-} \pi^{+}\right) \pi^{-}$ & 7.1 & 800 \\
$\circ \ell+$ jets & & 3.0 & 570 \\
$\circ$ fully hadronic & $\bar{B}_{s}^{0} \rightarrow \bar{D}^{0}\left(K^{-} \pi^{+}\right) K^{-} \pi^{+}$ & 1.6 & 200 \\
$\circ \ell+$ jets & & 0.7 & 3800 \\
Sum of all channels & & 12.4 & 11000 \\
\hline TOTAL & & 33 & \\
\hline
\end{tabular}

Table 9. Expected number of $t \rightarrow \bar{B}_{(s)}^{0}+$ jet events in selected $t \bar{t}$ final-states (fully hadronic, and $\ell+$ jets) with $\bar{B}_{(s)}^{0}$-meson decays into a $D$-meson plus 1 or 2 light mesons, after estimating branching ratios and detector acceptance losses, in pp collisions at the LHC and FCC.

\section{Measurement of $t \rightarrow b($ jet $)+c($ jet $)$ in pp collisions at the LHC and FCC}

Given the large signal reduction factors involved in the exclusive $\bar{B}_{(s)}^{0}$ hadronic final states discussed in the previous section, we can try an observation of the two-body $t \rightarrow \bar{B}_{(s)}^{0}+c$ decay minimizing the $\bar{B}_{(s)}^{0}$ decay branching fraction losses, by using events with fullyreconstructed bottom and charm jets. Namely, by reconstructing the dijet final state $t \rightarrow j$ (charm) $+\mathrm{j}$ (bottom), where the bottom jet is obtained clustering all products from the $\bar{B}_{(s)}^{0}$ decay (plus any other low- $p_{\mathrm{T}}$ hadron from any soft gluon potentially emitted previously from the parent $b$ quark), and where both heavy-quark jets have an invariant $m_{b, c}$ mass consistent with $m_{t}$. Such a final state is particularly attractive as it represents the decay of a top quark into two jets corresponding to the consecutively lighter (bottom and charm) quark families. The alternative bottom-plus-light-quark dijet final state, from the $t \rightarrow \bar{B}_{(s)}^{0}+u$ decay, should be much more swamped by the large QCD multijet background and will not be further considered. To reduce multijet combinatorial backgrounds, the 
analysis is carried out in $t \bar{t}$ lepton+jets events that pass the trigger selection discussed in section 3.1.2. In more detail, the final state of interest for this search is an event with one bottom and one charm jet (from the 2-body top decay of concern), plus an extra bottom jet and a high $-p_{\mathrm{T}}$ isolated lepton from the decay of the other accompanying top quark. The following baseline event selection is considered. Events are required to have:

1. Exactly 3 jets tagged as coming from two bottom and one charm quarks respectively (with the tagging performances discussed below), with at least one $b$ - plus $c$-jet pair having an invariant mass $120 \leq m_{b, c} \leq 220 \mathrm{GeV}$ and azimuthal separation $\Delta \phi(b, c)>$ $0.5 \pi$. Jets are reconstructed with the anti- $k_{T}$ algorithm with $R=0.5, p_{\mathrm{T}}>25 \mathrm{GeV}$, and $|\eta|<3.0$ (5.0) at 14 (100) TeV. In order to suppress backgrounds, and following the decay kinematics properties of the signal events discussed at the beginning of section 2 , we further require the tagged $c$-jet to be boosted $\left(p_{T}>m_{t} / 2 \approx 80 \mathrm{GeV}\right)$.

2. An isolated $\left(R_{\text {isol }}=0.3\right)$ charged lepton $\ell$ is required with $p_{\mathrm{T}}>30 \mathrm{GeV}$ and $|\eta|<2.5$ (5.0) at $14(100) \mathrm{TeV}$. Isolation is defined at the particle level, by requiring that the scalar sum of the $p_{\mathrm{T}}$ of all charged and neutral particles within $R_{\text {isol }}$ (except the charged lepton and any neutrinos) is less than $15 \%$ of the muon or electron $p_{\mathrm{T}}$.

The following bottom- and charm-jets reconstruction performances are assumed based on existing heavy-flavour studies in $t \bar{t}$ events at the LHC [70]:

- Bottom-quark jets: $b$-jet tagging efficiency: $75 \%, b$-jet mistagging probability for a $c$-quark: $5 \%$, and $b$-jet mistagging probability for $u d s g$ (light quarks or gluon): $0.5 \%$.

- Charm-quark jets: two different $c$-jet working points are considered: (i) $c$-jet tagging efficiency: $65 \%, c$-jet mistagging probability for a $b$ quark: $10 \%$, and $c$-jet mistagging probability for udsg: $10 \%$ [labeled 'HcT' hereafter]; and (ii) $c$-jet tagging efficiency: $35 \%, c$-jet mistagging probability for a $b$-quark: $5 \%$, and $c$-jet mistagging probability for udsg: $1 \%$ ['LcT' label].

The analysis criteria listed above are implemented into a NLO MG5_AMC simulation using the NNPDF30_nlo_as_0118 PDF and PYTHIA 8 for the parton shower (PS) and hadronization, with the PS matched to the matrix elements via the MC@NLO prescription [71]. The underlying event in pp collisions, of relevance for a realistic application of the lepton isolation criterion listed above, is generated with the 2013 Monash tune [72]. The signal NLO cross sections are scaled to NNLO+NNLL accuracy by multiplying them by a $K=1.2$ factor [35]. The use of an overall scaling, rather than of fully differential NNLO cross sections, is a standard procedure in the analysis of $t \bar{t}$ cross sections at the LHC. This is fully justified given that, as shown in [73], the ratio of NNLO over NLO differential distributions of $t \bar{t}$ cross sections at the LHC is basically flat as a function of top quark transverse momentum and rapidity, apart from such a global overall normalization. Background samples are simulated with the same setup for the following processes that can produce similar final states: standard-decay $t \bar{t}$; single top $(t+W, t+q$, and $t+b)$; 


\begin{tabular}{|l|cc|cc|}
\hline \multirow{2}{*}{ Selection criteria } & \multicolumn{2}{|c|}{ pp at $\sqrt{s}=14 \mathrm{TeV}\left(3 \mathrm{ab}^{-1}\right)$} & \multicolumn{2}{|c|}{ pp at $\sqrt{s}=100 \mathrm{TeV}\left(20 \mathrm{ab}^{-1}\right)$} \\
& 'HcT' & 'LcT' & 'HcT' & 'LcT' \\
\hline$(0):$ none & $1.0 \cdot 10^{9}$ & $1.0 \cdot 10^{9}$ & $2.4 \cdot 10^{11}$ & $2.4 \cdot 10^{11}$ \\
$(1):$ trigger $(\ell+$ jets $)$ & $4.8 \cdot 10^{8}$ & $4.8 \cdot 10^{8}$ & $1.3 \cdot 10^{11}$ & $1.3 \cdot 10^{11}$ \\
$(2):(1)+\left(N_{j_{b}}=2 \& \& N_{j_{c}}=1\right)$ & $8.2 \cdot 10^{7}$ & $3.8 \cdot 10^{7}$ & $2.3 \cdot 10^{10}$ & $1.2 \cdot 10^{10}$ \\
$(3):(1)+(2)+120 \leq m_{b, c} \leq 220 \mathrm{GeV}$ & $5.3 \cdot 10^{7}$ & $2.6 \cdot 10^{7}$ & $1.3 \cdot 10^{10}$ & $7.3 \cdot 10^{9}$ \\
\hline
\end{tabular}

Table 10. Total number of events expected for $t \bar{t} \rightarrow N_{j}+\ell^{ \pm}+X$ with $\ell^{ \pm}=\mu^{ \pm}, e^{ \pm}$, in pp collisions at $\sqrt{s}=14$ and $100 \mathrm{TeV}$, passing the analysis criteria for the 'HcT' and 'LcT' charm jet reconstruction performances discussed in the text. The "trigger" row lists the number of events that pass the trigger cuts presented in subsection 3.1.2, and $\left(N_{j_{b}}=2 \& \& N_{j_{c}}=1\right)$ indicates the exact requirement of two $b$ - and one $c$-quark jets reconstructed in the event with the kinematic criteria discussed in the text.

\begin{tabular}{|l|cc|cc|}
\hline \multirow{2}{*}{ process } & \multicolumn{2}{|c|}{$\begin{array}{c}\text { pp at } \sqrt{s}=14 \mathrm{TeV}\left(3 \mathrm{ab}^{-1}\right) \\
\text { 'HcT' }\end{array}$} & 'LcT' & pp at $\sqrt{s}=100 \mathrm{TeV}\left(20 \mathrm{ab}^{-1}\right)$ \\
& 'HcT' & 'LcT' \\
\hline$t \bar{t}$ (signal) & 5300 & 2500 & $1.4 \cdot 10^{6}$ & $7.5 \cdot 10^{5}$ \\
$t \bar{t}$ (backgd.) & $2.1(1.7) \cdot 10^{6}$ & $9.3(7.4) \cdot 10^{5}$ & $3.0(2.4) \cdot 10^{8}$ & $1.1(0.9) \cdot 10^{8}$ \\
$t W$ & $7.0(5.8) \cdot 10^{4}$ & $3.1(2.5) \cdot 10^{4}$ & $1.2(1.0) \cdot 10^{7}$ & $4.7(4.4) \cdot 10^{6}$ \\
$t+$ jet & $7.8(6.6) \cdot 10^{4}$ & $2.7(2.4) \cdot 10^{4}$ & $1.1(1.0) \cdot 10^{7}$ & $4.0(3.7) \cdot 10^{6}$ \\
$V+$ jet & $1.5(1.3) \cdot 10^{5}$ & $7.6(7.6) \cdot 10^{4}$ & $1.1(0.8) \cdot 10^{7}$ & $1.1(0.8) \cdot 10^{7}$ \\
$V V$ & $7.7(6.3) \cdot 10^{3}$ & $2.8(2.3) \cdot 10^{3}$ & $9.1(7.3) \cdot 10^{5}$ & $3.4(3.1) \cdot 10^{5}$ \\
Multijet & $<4.8 \cdot 10^{4}$ & $<4.8 \cdot 10^{4}$ & $<1.2 \cdot 10^{7}$ & $<1.2 \cdot 10^{7}$ \\
\hline $\mathcal{S}$ (std. dev.) & 6.1 & 4.5 & 130. & 110. \\
\hline
\end{tabular}

Table 11. Total number of bottom+charm jet pairs (events, in parentheses) expected over $m_{b, c}=$ $120-220 \mathrm{GeV}$ for signal and backgrounds after all analysis cuts (see text), in lepton+jets $t \bar{t}$ final states simulated in pp collisions at $\sqrt{s}=14$ and $100 \mathrm{TeV}$, for the 'HcT' and 'LcT' charm jet reconstruction performances described in the text. The last row lists the expected signal significance $\mathcal{S}$ (in standard deviations) for the top-quark 2-body decay reconstructed as $t \rightarrow b$-jet $+c$-jet.

$W+$ jets $(W+u d s g, W+c, W+b)$ and $Z+$ jets $(Z+g, Z+c, Z+b)$ collectively labeled $V+$ jet; dibosons $(V V=W W, Z Z, W Z)$; and QCD multijets $(b \bar{b}+X, c \bar{c}+X, q \bar{q}+X){ }^{6}$

After full jet reconstruction and implementing the bottom and charm jet (mis)identification performances and basic cuts listed above, we obtain the $t \bar{t}$ yields listed in table 10 after each one of the analysis steps. The final numbers after cuts are listed in table 11 for signal and each one of the individual backgrounds.

The expected number of signal $t \bar{t}$ lepton+jets events where one top quark decays into a reconstructed pair of bottom and charm jets after cuts is $N_{S}=5300$ and 1.4 million in pp collisions at the LHC and FCC, respectively, for the 'HcT' selection. The numbers for the more stringent ' $\mathrm{LcT}$ ' charm reconstruction performance are about twice smaller.

\footnotetext{
${ }^{6}$ Full-NLO+PS multijet events are very heavy to produce in terms of computing time, and are generated here based on LO+PS simulations alone.
} 

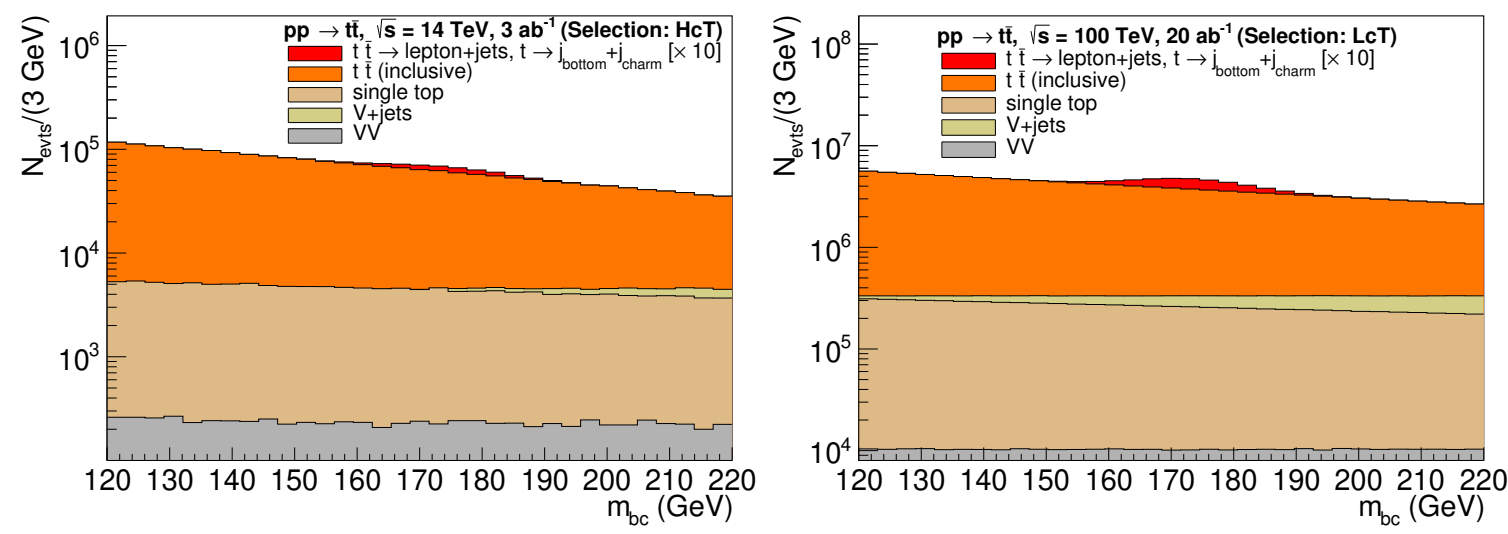

Figure 2. Expected invariant mass distributions for pairs of charm and bottom jets reconstructed passing the analysis criteria for the $t \rightarrow b c$ analysis simulated for signal and backgrounds in pp collisions at $\sqrt{s}=14 \mathrm{TeV}$ (left) and $100 \mathrm{TeV}$ (right) for the 'HcT' and 'LcT' charm jet reconstruction performances, respectively, described in the text. The different histograms show the Gaussian signal (red, scaled by a factor of 10 for visibility) and individual (exponential-like) backgrounds, stacked one above each other, listed in table 11.

The applied selection criteria reduce the produced number of $t \rightarrow \bar{B}_{(s)}^{0}+c$ signal events by about a factor of six (ten) at the LHC (FCC), while reducing the backgrounds by two to three orders of magnitude. The most important remaining background passing the analysis cuts is that from $t \bar{t}$ production with standard top-quarks decays, which have a final expected number of events of about $N_{B}=0.7-1.7$ and 90-240 millions at the LHC and FCC respectively, that are about one order of magnitude larger than the sum of the rest of backgrounds. ${ }^{7}$ Representative dijet mass distributions are shown in figure 2 left (right) after all cuts, for the 'HcT' ('LcT') selections at the LHC (FCC). The signal statistical significances are computed from the number of signal pairs over the square-root of the sum of all backgrounds pairs, $\mathcal{S}=N_{S} / \sqrt{N_{B}}$, in a window of 1.4 times the width of the reconstructed top mass, where we assume a dijet invariant mass resolution of $\delta m_{b, c}=$ $9 \mathrm{GeV}$, which thereby translates into a significance calculated over the $m_{b, c}=160-190 \mathrm{GeV}$ mass range. No uncertainty in the background is assumed, as the continuum can be very accurately measured and fitted to a smooth function from the measured off-peak dijet mass distributions. The observation of the top-quark 2-body decay in the bottom+charm dijet final state is warranted both at the HL-LHC and FCC, as indicated by the $\mathcal{S}>5$ std. dev. results listed in the last row of table 11. More advanced profile-likelihood statistical analyses for signal and backgrounds, typical of standard LHC analyses that go beyond the scope of this first study, can easily improve the simple significance estimates presented here that should thereby be considered as conservative lower limits.

\footnotetext{
${ }^{7}$ The simulation of the QCD multijet backgrounds is very time-consuming, and only an upper limit in the number of events passing the analysis cuts can be provided from the zero events selected out of 10 million generated. In the real data analysis, those events will be well understood using control regions with appropriately reversed ("anti-signal") cuts.
} 

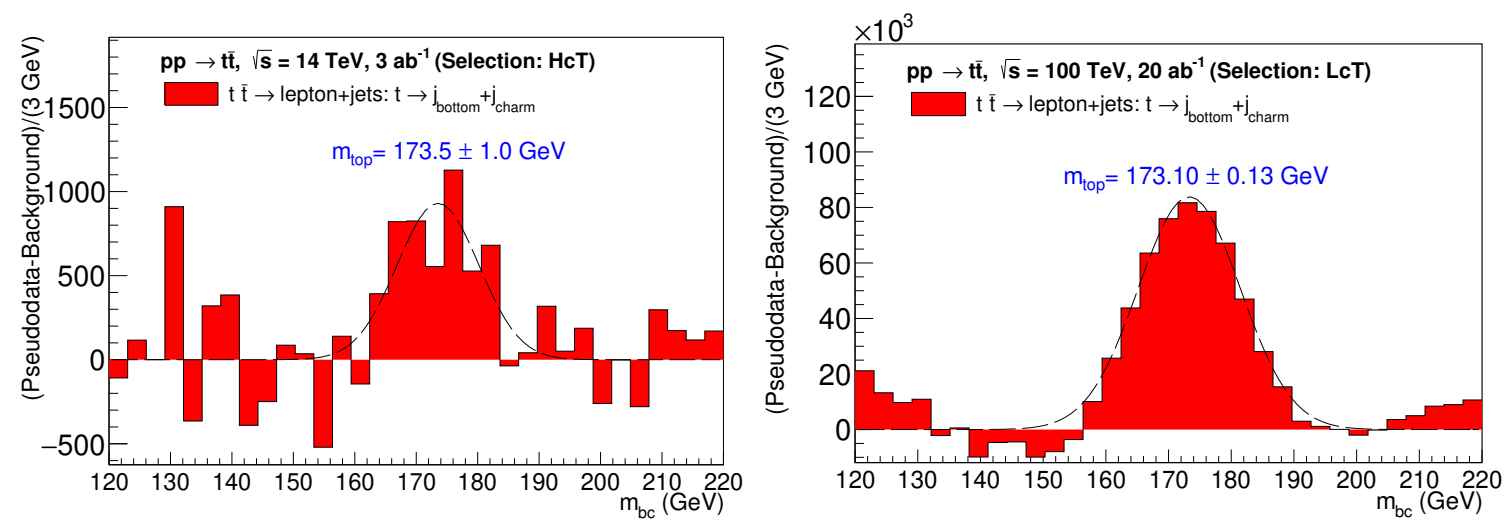

Figure 3. Expected background-subtracted invariant mass distributions for pairs of charm and bottom jets reconstructed in the $t \rightarrow b c$ analysis in simulated pp collisions at $\sqrt{s}=14 \mathrm{TeV}$ (left) and $100 \mathrm{TeV}$ (right) after cuts for the 'HcT' and 'LcT' charm jet reconstruction performances, respectively, discussed in the text. The dashed line shows a Gaussian fit to the distributions, with the fitted value of the top-quark mass listed above them.

\subsection{Top-quark mass via bottom+charm dijet decays}

The expected bottom-charm dijet invariant mass distributions after analysis cuts plotted in figure 2 show a Gaussian-like signal peak, with a width driven by the experimental $\delta m_{b, c}=9 \mathrm{GeV}$ dijet mass resolution assumed here, on top of the sum of all continuum backgrounds with an overall exponential- or powerlaw-like smooth shape. By fitting the $m_{b, c}$ distribution to an exponential-type function outside of the peak, one can estimate the expected background contributions below the top mass peak. The resulting 2-body top-quark signal mass distributions, with the fitted background subtracted, are plotted in figure 3 for pp collisions at $\sqrt{s}=14 \mathrm{TeV}$ (left) and $100 \mathrm{TeV}$ (right) for the 'HcT' and 'LcT' charm jet reconstruction performances, respectively. The values of the reconstructed top-quark mass and associated uncertainties quoted in the plots are those obtained from the corresponding Gaussian fits, obtained with a $\chi^{2}$ method, shown by the dashed lines in figure 3. Running multiple pseudoexperiments that generate the expected dijet mass distributions for signal and backgrounds, we derive fit uncertainties in the top-quark mass of the order of $1 \mathrm{GeV}$ at the LHC and $0.15 \mathrm{GeV}$ at the FCC, listed in the figure. Beyond such uncertainties, statistical in nature, one would need to consider systematic uncertainties from the jet energy scale calibration that propagate into the dijet mass peak position. Nonetheless, with millions heavy-flavour jets reconstructed in dedicated studies in this energy range, the latter uncertainties should be well under control (and, in any case, would be smaller than in the standard $t \rightarrow b \bar{q} q^{\prime}$ triple-jet final state). More advanced combined signal+background fit procedures exist, standard now in similar analyses at the LHC [74], that would provide a best-fit top-quark mass with potentially smaller uncertainties than those quoted here. We leave this more detailed work for an upcoming study. The top-quark mass determination presented here has systematic uncertainties different (and, in some cases, smaller) than other extractions considered so far based on different kinematic fits of the decay products to a particular $t \bar{t}$ decay hypothesis [74]. On the one hand, our reconstructed final state has 


\begin{tabular}{|c|c|c|c|c|c|}
\hline \multirow[t]{2}{*}{$\begin{array}{l}\text { pp system } \\
\sqrt{s}, \mathcal{L}_{\text {int }}\end{array}$} & \multicolumn{2}{|c|}{$\begin{array}{l}\text { Number of produced } \\
\text { events expected }\end{array}$} & \multicolumn{3}{|c|}{$\begin{array}{l}\text { Number of } 2 \text {-body top-quark events } \\
\text { after } \mathcal{B} \times \text { acceptance } \times \text { efficiency cuts }\end{array}$} \\
\hline & $t \bar{t}$ & $t \rightarrow \bar{B}_{(s)}^{0}+$ jet & $\bar{B}_{(s)}^{0} \rightarrow J / \psi h h$ & $\bar{B}_{(s)}^{0} \rightarrow D_{(s)}^{0, \pm} h(h)$ & $t \rightarrow b+c$ \\
\hline $14 \mathrm{TeV}, 3 \mathrm{ab}^{-1}$ & $3 \cdot 10^{9}$ & $2.5 \cdot 10^{5}$ & 16 & 33 & 5300 \\
\hline $100 \mathrm{TeV}, 20 \mathrm{ab}^{-1}$ & $7 \cdot 10^{11}$ & $6 \cdot 10^{7}$ & 5200 & 11000 & $1.4 \cdot 10^{6}$ \\
\hline
\end{tabular}

Table 12. Summary of the total number of top-quark events expected in the various final states considered in this work for pp collisions at $\sqrt{s}=14 \mathrm{TeV}\left(3 \mathrm{ab}^{-1}\right)$ and $100 \mathrm{TeV}\left(20 \mathrm{ab}^{-1}\right)$. The first two columns list the number of produced pair $t \bar{t}$ and two-body $t$-quark decay events expected. The last three columns list the number of 2-body $t \bar{t} \rightarrow\left(\bar{B}_{s}^{0}+c, u\right)+X$ top-quarks events expected in measurements using $\bar{B}_{(s)}^{0} \rightarrow J / \psi h h$ and $\bar{B}_{(s)}^{0} \rightarrow D^{0, \pm} h(h)$ with $h=\pi, K$, and $t \rightarrow j_{b}+j_{c}$ final states, after accounting for their respective branching fractions, acceptance, and reconstruction efficiencies.

smaller jet multiplicities (and thereby smaller propagated jet energy scale uncertainties) and, on the other, we do not need to use additional decay lepton information. Combining the top-quark mass measurement from the two-body charm+bottom dijet final state with those derived from other methods would thereby allow for a more accurate and precise extraction of this key SM parameter.

\section{Summary}

Rare two-body decays of the top quark into a neutral $B$-meson plus an up- or charmquark jet, $t \rightarrow \bar{B}^{0}+u, c$ and $t \rightarrow \bar{B}_{s}^{0}+c, u$, as well as into a bottomonium meson plus a jet $t \rightarrow \Upsilon(n S)+c, u$, have been studied for the first time. The leading-order branching ratios have been computed in a non-relativistic quantum chromodynamics (NRQCD) quark recombination model, and amount to $\mathcal{B}\left(t \rightarrow \bar{B}_{(s)}^{0}+\right.$ jet $) \approx 4 \cdot 10^{-5}$, and $\mathcal{B}(t \rightarrow \Upsilon(n S)+$ jet $) \approx 2.5 \cdot 10^{-9}$. The feasibility of an experimental observation of such decays in multiple final states has been studied in $t \bar{t}$ pair production events in proton-proton collisions at $\sqrt{s}=14 \mathrm{TeV}$ at the HL-LHC and at $100 \mathrm{TeV}$ at the FCC with integrated luminosities of $3 \mathrm{ab}^{-1}$ and $20 \mathrm{ab}^{-1}$ respectively. For each one of the final states considered, we take into account realistic estimates for their acceptance and reconstruction efficiencies based on current LHC studies. Table 12 summarizes the expected number of events in various decay final-state channels considered. Combining the results from all exclusive $\bar{B}_{(s)}^{0}$ decays, one expects a grand total of about 50 (16000) two-body top-quark decays reconstructed at the LHC (FCC) in the $J / \psi h h$ and $D^{0, \pm} h(h)$ final states (with $h=\pi, K$ ), respectively. The expected numbers of top-quark 2-body decays, $t \rightarrow \bar{B}_{(s)}^{0}+c$, where the final state is reconstructed as a bottom-plus-charm dijet system are 5300 and 1.4 million after cuts in pp collisions at the LHC and FCC, respectively. A clear observation above backgrounds of the top-quark 2-body decay in such intriguing bottom+charm dijet final state appears warranted both at the LHC and FCC.

We have also estimated the possibility to use the $t \rightarrow j_{b}+j_{c}$ final state to determine the top-quark mass via a simple dijet invariant mass analysis. Gaussian fit mass uncertainties of the order of $1 \mathrm{GeV}$ at the LHC and $0.15 \mathrm{GeV}$ at the FCC are expected. Such a top- 
quark mass determination, with systematic uncertainties smaller and different than other extractions considered so far, would allow for a more accurate and precise extraction of this key parameter of the Standard Model.

\section{Acknowledgments}

We are thankful to Pedro da Silva and Adish Vartak for helpful discussions on top-quark measurements and dijet resonances fitting procedures at the LHC, respectively, and to Michelangelo Mangano for useful comments. The work of HSS is supported by the ILP Labex (ANR-11-IDEX-0004-02, ANR-10-LABX-63).

Open Access. This article is distributed under the terms of the Creative Commons Attribution License (CC-BY 4.0), which permits any use, distribution and reproduction in any medium, provided the original author(s) and source are credited.

\section{References}

[1] Particle Data Group collaboration, Review of particle physics, Phys. Rev. D 98 (2018) 030001 [INSPIRE].

[2] S. Alekhin, A. Djouadi and S. Moch, The top quark and Higgs boson masses and the stability of the electroweak vacuum, Phys. Lett. B 716 (2012) 214 [arXiv:1207.0980] [INSPIRE].

[3] G. Apollinari, O. Brüning, T. Nakamoto and L. Rossi, High Luminosity Large Hadron Collider HL-LHC, CERN Yellow Rep. (2015) 1 [arXiv: 1705.08830] [INSPIRE].

[4] FCC collaboration, FCC-hh: the hadron collider. Future Circular Collider conceptual design report volume 3, Eur. Phys. J. ST 228 (2019) 755 [INSPIRE].

[5] M.L. Mangano et al., Physics at a 100 TeV pp collider: Standard Model processes, CERN Yellow Rep. (2017) 1 [arXiv:1607.01831] [INSPIRE].

[6] P. Azzi and M. Cristinziani, Top quark physics at the High Luminosity LHC, in $9^{\text {th }}$ International Workshop on Top Quark Physics, (2017) [arXiv:1702.04598] [INSPIRE].

[7] E. Braaten, Y. Jia and T. Mehen, B production asymmetries in perturbative QCD, Phys. Rev. D 66 (2002) 034003 [hep-ph/0108201] [INSPIRE].

[8] G.T. Bodwin, E. Braaten and G. Lepage, Rigorous QCD analysis of inclusive annihilation and production of heavy quarkonium, Phys. Rev. D 51 (1995) 1125 [Erratum ibid. 55 (1997) 5853] [hep-ph/9407339] [INSPIRE].

[9] C.-H. Chang, J.-X. Wang and X.-G. Wu, Production of $B_{c}$ or $\bar{B}_{c}$ meson and its excited states via $\bar{t}$ quark or $t$ quark decays, Phys. Rev. D 77 (2008) 014022 [arXiv:0711.1898] [INSPIRE].

[10] A.V. Berezhnoy, V.V. Kiselev and A.K. Likhoded, New insight into the photoproduction of $D^{*}$ meson in $Q C D$, hep-ph/9901333 [INSPIRE].

[11] H.-S. Shao and Y.-J. Zhang, Complete study of hadroproduction of a $\Upsilon$ meson associated with a prompt $J / \psi$, Phys. Rev. Lett. 117 (2016) 062001 [arXiv: 1605.03061] [INSPIRE].

[12] M. Krämer, Quarkonium production at high-energy colliders, Prog. Part. Nucl. Phys. 47 (2001) 141 [hep-ph/0106120] [INSPIRE]. 
[13] R. Sharma and I. Vitev, High transverse momentum quarkonium production and dissociation in heavy ion collisions, Phys. Rev. C 87 (2013) 044905 [arXiv: 1203.0329] [InSPIRE].

[14] H. Han, Y.-Q. Ma, C. Meng, H.-S. Shao, Y.-J. Zhang and K.-T. Chao, $\Upsilon(n S)$ and $\chi_{b}(n P)$ production at hadron colliders in nonrelativistic QCD, Phys. Rev. D 94 (2016) 014028 [arXiv: 1410.8537] [INSPIRE].

[15] Y. Feng, B. Gong, L.-P. Wan and J.-X. Wang, An updated study of $\Upsilon$ production and polarization at the Tevatron and LHC, Chin. Phys. C 39 (2015) 123102 [arXiv:1503.08439] [INSPIRE].

[16] CMS collaboration, Top FCNC searches at HL-LHC with the CMS experiment, EPJ Web Conf. 191 (2018) 02009 [arXiv: 1808.09915] [INSPIRE].

[17] LHCb collaboration, Measurement of the CP-violating phase $\phi_{s}$ in the decay $B_{s}^{0} \rightarrow J / \psi \phi$, Phys. Rev. Lett. 108 (2012) 101803 [arXiv:1112.3183] [inSPIRE].

[18] LHCb collaboration, Observation of $B_{s} \rightarrow J / \psi f_{2}^{\prime}(1525)$ in $J / \psi K^{+} K^{-}$final states, Phys. Rev. Lett. 108 (2012) 151801 [arXiv:1112.4695] [INSPIRE].

[19] LHCb collaboration, Analysis of the resonant components in $B_{s} \rightarrow J / \psi \pi^{+} \pi^{-}$, Phys. Rev. D 86 (2012) 052006 [arXiv: 1204.5643] [INSPIRE].

[20] LHCb collaboration, Measurement of B meson production cross-sections in proton-proton collisions at $\sqrt{s}=7 \mathrm{TeV}$, JHEP 08 (2013) 117 [arXiv: 1306.3663] [INSPIRE].

[21] LHCb collaboration, Amplitude analysis and the branching fraction measurement of $\bar{B}_{s}^{0} \rightarrow J / \psi K^{+} K^{-}$, Phys. Rev. D 87 (2013) 072004 [arXiv:1302.1213] [INSPIRE].

[22] LHCb collaboration, Analysis of the resonant components in $B^{-}+0 \rightarrow J / \psi \pi^{+} \pi^{-}$, Phys. Rev. D 87 (2013) 052001 [arXiv:1301.5347] [INSPIRE].

[23] CMS and LHCb collaborations, Observation of the rare $B_{s}^{0} \rightarrow \mu^{+} \mu^{-}$decay from the combined analysis of CMS and LHCb data, Nature $\mathbf{5 2 2}$ (2015) 68 [arXiv:1411.4413] [INSPIRE].

[24] LHCb collaboration, Study of the production of $\Lambda_{b}^{0}$ and $\bar{B}^{0}$ hadrons in pp collisions and first measurement of the $\Lambda_{b}^{0} \rightarrow J / \psi p K^{-}$branching fraction, Chin. Phys. C 40 (2016) 011001 [arXiv: 1509.00292] [INSPIRE].

[25] LHCb collaboration, Measurement of b-hadron production fractions in $7 \mathrm{TeV}$ pp collisions, Phys. Rev. D 85 (2012) 032008 [arXiv:1111.2357] [INSPIRE].

[26] LHCb collaboration, Observation of $B^{0} \rightarrow \bar{D}^{0} K^{+} K^{-}$and evidence of $B_{s}^{0} \rightarrow \bar{D}^{0} K^{+} K^{-}$, Phys. Rev. Lett. 109 (2012) 131801 [arXiv:1207.5991] [INSPIRE].

[27] LHCb collaboration, Observation of the decay $B_{s}^{0} \rightarrow \bar{D}^{0} \phi$, Phys. Lett. B 727 (2013) 403 [arXiv: 1308.4583] [INSPIRE].

[28] LHCb collaboration, Measurement of the branching fractions of the decays $B_{s}^{0} \rightarrow \bar{D}^{0} K^{-} \pi^{+}$ and $B^{0} \rightarrow \bar{D}^{0} K^{+} \pi^{-}$, Phys. Rev. D 87 (2013) 112009 [arXiv: 1304.6317] [INSPIRE].

[29] LHCb collaboration, Dalitz plot analysis of $B^{0} \rightarrow \bar{D}^{0} \pi^{+} \pi^{-}$decays, Phys. Rev. D 92 (2015) 032002 [arXiv: 1505.01710] [INSPIRE].

[30] LHCb collaboration, Observation of $B_{s}^{0} \rightarrow \bar{D}^{* 0} \phi$ and search for $B^{0} \rightarrow \bar{D}^{0} \phi$ decays, Phys. Rev. D 98 (2018) 071103 [arXiv: 1807.01892] [INSPIRE]. 
[31] LHCb collaboration, Observation of the decay $B_{s}^{0} \rightarrow \bar{D}^{0} K^{+} K^{-}$, Phys. Rev. D 98 (2018) 072006 [arXiv: 1807.01891] [INSPIRE].

[32] LHCb collaboration, Measurement of $B^{+}, B^{0}$ and $\Lambda_{b}^{0}$ production in $p P b$ collisions at $\sqrt{s_{\mathrm{NN}}}=8.16 \mathrm{TeV}$, Phys. Rev. D 99 (2019) 052011 [arXiv: 1902.05599] [inSPIRE].

[33] LHCb collaboration, First observation of top quark production in the forward region, Phys. Rev. Lett. 115 (2015) 112001 [arXiv:1506.00903] [INSPIRE].

[34] LHCb collaboration, Measurement of forward top pair production in the dilepton channel in pp collisions at $\sqrt{s}=13 \mathrm{TeV}$, JHEP 08 (2018) 174 [arXiv: 1803.05188] [INSPIRE].

[35] M. Czakon, P. Fiedler and A. Mitov, Total top-quark pair-production cross section at hadron colliders through $O\left(\alpha_{S}^{4}\right)$, Phys. Rev. Lett. 110 (2013) 252004 [arXiv: 1303.6254] [INSPIRE].

[36] NNPDF collaboration, Parton distributions for the LHC run II, JHEP 04 (2015) 040 [arXiv: 1410.8849] [INSPIRE].

[37] ATLAS collaboration, Measurement of the top-quark mass in the fully hadronic decay channel from ATLAS data at $\sqrt{s}=7$ TeV, Eur. Phys. J. C $\mathbf{7 5}$ (2015) 158 [arXiv: 1409.0832] [INSPIRE].

[38] ATLAS collaboration, Top-quark mass measurement in the all-hadronic t $\bar{t}$ decay channel at $\sqrt{s}=8 \mathrm{TeV}$ with the ATLAS detector, JHEP 09 (2017) 118 [arXiv:1702.07546] [INSPIRE].

[39] ATLAS collaboration, Measurements of t $\bar{t}$ differential cross-sections of highly boosted top quarks decaying to all-hadronic final states in pp collisions at $\sqrt{s}=13 \mathrm{TeV}$ using the ATLAS detector, Phys. Rev. D 98 (2018) 012003 [arXiv:1801.02052] [INSPIRE].

[40] CMS collaboration, Measurement of the top-quark mass in all-jets t $\bar{t}$ events in pp collisions at $\sqrt{s}=7 \mathrm{TeV}$, Eur. Phys. J. C $\mathbf{7 4}$ (2014) 2758 [arXiv:1307.4617] [INSPIRE].

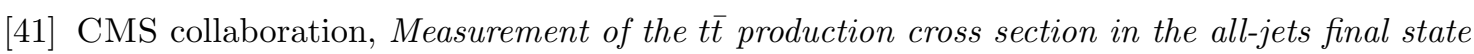
in pp collisions at $\sqrt{s}=8 \mathrm{TeV}$, Eur. Phys. J. C $\mathbf{7 6}$ (2016) 128 [arXiv:1509.06076] [INSPIRE].

[42] CMS collaboration, Measurement of the top quark mass in the all-jets final state at $\sqrt{s}=13 \mathrm{TeV}$ and combination with the lepton+jets channel, Eur. Phys. J. C 79 (2019) 313 [arXiv: 1812.10534] [INSPIRE].

[43] M. Cacciari, G.P. Salam and G. Soyez, The anti- $k_{t}$ jet clustering algorithm, JHEP 04 (2008) 063 [arXiv: 0802.1189] [INSPIRE].

[44] M. Cacciari, G.P. Salam and G. Soyez, FastJet user manual, Eur. Phys. J. C 72 (2012) 1896 [arXiv:1111.6097] [INSPIRE].

[45] J. Alwall et al., The automated computation of tree-level and next-to-leading order differential cross sections and their matching to parton shower simulations, JHEP 07 (2014) 079 [arXiv: 1405.0301] [INSPIRE].

[46] CMS collaboration, Measurement of the top-antitop production cross section in pp collisions at $\sqrt{s}=7 \mathrm{TeV}$ using the kinematic properties of events with leptons and jets, Eur. Phys. J. C 71 (2011) 1721 [arXiv:1106.0902] [INSPIRE].

[47] ATLAS collaboration, Measurement of the top quark pair production cross-section with ATLAS in the single lepton channel, Phys. Lett. B 711 (2012) 244 [arXiv:1201.1889] [INSPIRE]. 
[48] ATLAS collaboration, Measurement of the top pair production cross section in $8 \mathrm{TeV}$ proton-proton collisions using kinematic information in the lepton+jets final state with ATLAS, Phys. Rev. D 91 (2015) 112013 [arXiv:1504.04251] [INSPIRE].

[49] CMS collaboration, Measurements of the t $\bar{t}$ production cross section in lepton+jets final states in pp collisions at $8 \mathrm{TeV}$ and ratio of 8 to $7 \mathrm{TeV}$ cross sections, Eur. Phys. J. C 77 (2017) 15 [arXiv: 1602.09024] [INSPIRE].

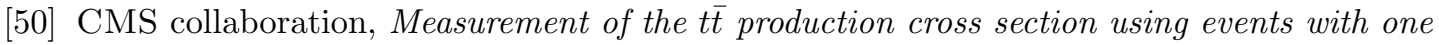
lepton and at least one jet in pp collisions at $\sqrt{s}=13 \mathrm{TeV}$, JHEP 09 (2017) 051 [arXiv: 1701.06228] [INSPIRE].

[51] ATLAS collaboration, Measurement of the inclusive and fiducial $t \bar{t}$ production cross-sections in the lepton+jets channel in pp collisions at $\sqrt{s}=8 \mathrm{TeV}$ with the ATLAS detector, Eur. Phys. J. C 78 (2018) 487 [arXiv:1712.06857] [INSPIRE].

[52] J.M. Campbell and R.K. Ellis, MCFM for the Tevatron and the LHC, arXiv:1007.3492 [INSPIRE].

[53] D. d'Enterria, Top-quark pair production cross sections at NNLO+NNLL in $p P b$ collisions at $\sqrt{s_{\mathrm{NN}}}=8.16 \mathrm{TeV}$, Nucl. Phys. A 982 (2019) 75 [arXiv:1706.09521] [INSPIRE].

[54] ATLAS collaboration, Measurement of the b-hadron production cross section using decays to $D^{*} \mu^{-} X$ final states in pp collisions at $\sqrt{s}=7$ TeV with the ATLAS detector, Nucl. Phys. B 864 (2012) 341 [arXiv:1206.3122] [INSPIRE].

[55] ATLAS collaboration, Time-dependent angular analysis of the decay $B_{s}^{0} \rightarrow J / \psi \phi$ and extraction of $\Delta \Gamma_{s}$ and the CP-violating weak phase $\phi_{s}$ by ATLAS, JHEP 12 (2012) 072 [arXiv: 1208.0572] [INSPIRE].

[56] ATLAS collaboration, Search for the decay $B_{s}^{0} \rightarrow \mu \mu$ with the ATLAS detector, Phys. Lett. $B \mathbf{7 1 3}$ (2012) 387 [arXiv:1204.0735] [INSPIRE].

[57] ATLAS collaboration, Measurement of $D^{* \pm}, D^{ \pm}$and $D_{s}^{ \pm}$meson production cross sections in pp collisions at $\sqrt{s}=7 \mathrm{TeV}$ with the ATLAS detector, Nucl. Phys. B 907 (2016) 717 [arXiv: 1512.02913] [INSPIRE].

[58] CMS collaboration, Measurement of the $B^{0}$ production cross section in pp collisions at $\sqrt{s}=7$ TeV, Phys. Rev. Lett. 106 (2011) 252001 [arXiv:1104.2892] [InSPIRE].

[59] CMS collaboration, Measurement of the strange B meson production cross section with $J / \psi$ $\phi$ decays in pp collisions at $\sqrt{s}=7$ TeV, Phys. Rev. D 84 (2011) 052008 [arXiv:1106.4048] [INSPIRE].

[60] CMS collaboration, Search for $B_{s}^{0} \rightarrow \mu^{+} \mu^{-}$and $B^{0} \rightarrow \mu^{+} \mu^{-}$decays, JHEP 04 (2012) 033 [arXiv: 1203.3976] [INSPIRE].

[61] CMS collaboration, Angular analysis and branching fraction measurement of the decay $B^{0} \rightarrow K^{* 0} \mu^{+} \mu^{-}$, Phys. Lett. B 727 (2013) 77 [arXiv: 1308.3409] [InSPIRE].

[62] CMS collaboration, Angular analysis of the decay $B^{0} \rightarrow K^{* 0} \mu^{+} \mu^{-}$from pp collisions at $\sqrt{s}=8 \mathrm{TeV}$, Phys. Lett. B 753 (2016) 424 [arXiv:1507.08126] [INSPIRE].

[63] CMS collaboration, Study of B meson production in $p+P b$ collisions at $\sqrt{s_{\mathrm{NN}}}=5.02 \mathrm{TeV}$ using exclusive hadronic decays, Phys. Rev. Lett. 116 (2016) 032301 [arXiv:1508.06678] [INSPIRE]. 
[64] CMS collaboration, Measurement of the top quark mass using charged particles in pp collisions at $\sqrt{s}=8 \mathrm{TeV}$, Phys. Rev. D 93 (2016) 092006 [arXiv:1603.06536] [InSPIRE].

[65] CMS collaboration, Measurement of $B_{s}^{0}$ meson production in pp and $\mathrm{PbPb}$ collisions at $\sqrt{s_{\mathrm{NN}}}=5.02 \mathrm{TeV}$, Phys. Lett. B 796 (2019) 168 [arXiv:1810.03022] [INSPIRE].

[66] CMS collaboration, Nuclear modification factor of $D^{0}$ mesons in $\mathrm{PbPb}$ collisions at $\sqrt{s_{\mathrm{NN}}}=5.02 \mathrm{TeV}$, Phys. Lett. B $\mathbf{7 8 2}$ (2018) 474 [arXiv: 1708.04962] [INSPIRE].

[67] CMS collaboration, Studies of beauty suppression via nonprompt $D^{0}$ mesons in $\mathrm{Pb}-\mathrm{Pb}$ collisions at $Q^{2}=4 \mathrm{GeV}^{2}$, Phys. Rev. Lett. 123 (2019) 022001 [arXiv:1810.11102] [INSPIRE].

[68] T. Sjöstrand et al., An introduction to PYTHIA 8.2, Comput. Phys. Commun. 191 (2015) 159 [arXiv: 1410.3012] [INSPIRE].

[69] HFLAV collaboration, Averages of b-hadron, c-hadron and $\tau$-lepton properties as of 2018, arXiv:1909.12524 [INSPIRE].

[70] CMS collaboration, Identification of heavy-flavour jets with the CMS detector in pp collisions at $13 \mathrm{TeV}, 2018$ JINST $13 \mathrm{P} 05011$ [arXiv: 1712.07158] [INSPIRE].

[71] S. Frixione and B.R. Webber, Matching NLO QCD computations and parton shower simulations, JHEP 06 (2002) 029 [hep-ph/0204244] [INSPIRE].

[72] P. Skands, S. Carrazza and J. Rojo, Tuning PYTHIA 8.1: the Monash 2013 tune, Eur. Phys. J. $C \mathbf{7 4}(2014) 3024$ [arXiv: 1404.5630] [InSPIRE].

[73] S. Catani, S. Devoto, M. Grazzini, S. Kallweit and J. Mazzitelli, Top-quark pair production at the LHC: fully differential QCD predictions at NNLO, JHEP 07 (2019) 100 [arXiv: 1906.06535] [INSPIRE].

[74] ATLAS, CDF, CMS and D0 collaborations, First combination of Tevatron and LHC measurements of the top-quark mass, arXiv:1403.4427 [INSPIRE]. 